\title{
FUNCTIONS OF EXTENDED CLASS IN THE THEORY OF FUNCTIONS OF SEVERAL COMPLEX VARIABLES
}

\author{
BY \\ STEFAN BERGMAN
}

1. Introduction. $B$-harmonic functions and the need for extension of this class. In order to develop a theory of functions of several complex variables, in particular along patterns similar to those used in the theory of a single variable, it is necessary to develop tools analogous to those which in the case of one variable lead so successfully to numerous important results.

The principle of hyperbolic measure and that of harmonic measure are two of the most powerful methods in the theory of one variable. The introduction of complex orthogonal functions and the method of the minimum integral provide a suitable replacement of the methods connected with the principle of hyperbolic measure; they yield, in the case of one variable, most of the results which can be obtained by applying the first of the principles mentioned above; since these methods do not use the Riemann mapping theorem, they can easily be extended to the case of several complex variables.

The generalization of methods which use the principle of harmonic measure seems to be more involved. These methods are based on the introduction of harmonic functions of two real variables into the theory of analytic functions of one complex variable and the use of certain properties of the former functions. In particular, the fact that to every continuous functions defined on the (sufficiently smooth) boundary curve of a domain $\left.{ }^{1}\right) \mathfrak{B}^{2}$ there exists a function, harmonic in $\mathfrak{B}^{2}$, and assuming the prescribed boundary values, together with the fact that this function can be represented in a comparatively simple form by means of an integral, plays a decisive role in many investigations of the theory of one variable.

If one attempts to generalize these methods to the case of several complex variables, one immediately realizes that the corresponding approach for several variables meets serious difficulties and leads to methods of investigation which in many respects are weaker and considerably less flexible than in the case of one variable. Let us analyze the reason for this situation.

The real or imaginary part of an analytic function of two complex variables is a $B$-harmonic function, that is, satisfies the four equations:

$$
\frac{\partial^{2} U}{\partial x_{1}^{2}}+\frac{\partial^{2} U}{\partial y_{1}^{2}}=0, \quad \frac{\partial^{2} U}{\partial x_{2}^{2}}+\frac{\partial^{2} U}{\partial y^{2}}=0,
$$

Presented to the Society, August 22,1946, under the title The "extended class" in the theory of functions of two complex variables; received by the editors April 14, 1947.

(1) A superscript attached to a symbol representing a point set indicates its dimensions. 


$$
\frac{\partial^{2} U}{\partial x_{1} \partial x_{2}}+\frac{\partial^{2} \cdot U}{\partial y_{1} \partial y_{2}}=0, \quad \frac{\partial^{2} U}{\partial x_{1} \partial y_{2}}-\frac{\partial^{2} U}{\partial x_{2} \partial y_{1}}=0 .
$$

Every $B$-harmonic function is naturally a harmonic function of four real variables. On the other hand, not every harmonic function of four real variables is $B$-harmonic. The boundary of a four-dimensional domain (domain of definition of a function of two complex variables) is a three-dimensional manifold $\left({ }^{2}\right)$. It does not, from the point of view of the theory of functions, play the same role as the boundary curve in the case of a single variable. True, every harmonic (and a fortiori every $B$-harmonic) function assumes the maximum and minimum of its values on the (three-dimensional) boundary, and therefore the maximum of the real and imaginary parts (or of the absolute value) of a function of two complex variables is assumed on the boundary. On the other hand, $B$-harmonic functions represent a sub-class of harmonic functions, and a $B$-harmonic function (and a fortiori an analytic function of two complex variables) is uniquely determined by its value on certain sub-sets of the three-dimensional boundary. For instance, in the case of a bicylinder( $\left(^{3}\right) B^{4}=\mathrm{E}\left[\left|z_{1}\right|<1,\left|z_{2}\right|<1\right]$ the Cauchy formula yields the values of an analytic function in $B^{4}$ in terms of its value on the two-dimensional surface $B^{2}=\mathrm{E}\left[\left|z_{1}\right|=1,\left|z_{2}\right|=1\right]$, while the boundary of $B^{4}$ is $b^{3}=\mathrm{E}\left[\left|z_{1}\right|<1,\left|z_{2}\right|=1\right]+\mathrm{E}\left[\left|z_{1}\right|=1,\left|z_{2}\right|<1\right]$. (See also Remark 1, p. 527.) From this formula it can easily be deduced that every analytic function assumes the maximum of its absolute value not merely on $b^{3}$ but indeed on the "distinguished boundary surface" $B^{2}$. This situation suggests that the distinguished boundary surface may assume from the point of view of the theory of functions of two variables the role of the boundary curve in the case of one variable. The analogy fails, however, in one important direction; to every real continuous function defined on the (sufficiently smooth) boundary curve of a (plane) domain, there exists a harmonic function which assumes the prescribed values. On the other hand, it is quite easy to see that to a given real function defined on the distinguished boundary surface $B^{2}$ of a bicylinder, there does not always exist a $B$-harmonic function defined in $B^{4}$ which assumes on $B^{2}$ the prescribed values. Indeed, from the theory of Fourier series in two variables, it follows immediately that to every continuous function defined on $B^{2}=E\left[\left|z_{1}\right|=1,\left|z_{2}\right|=1\right]$ there exists and is uniquely determined by the values on $B^{2}$ a function which satisfies (1.1); but in general such a function will not satisfy in addition to (1.1) the system (1.2). (See Remark 2, p. 528).

(2) For simplicity's sake, we shall limit ourselves to the consideration of functions of two complex variables. We wish, however, to stress that the generalization of considerations to the case of $n$ complex variables is in almost all instances a question which involves only technical difficulties.

(3) See notation at the end of this section. 
The means of remedy could proceed in two directions. Either we can introduce subsets of $B^{2}$, such that to every real continuous function defined on such a subset there exists one and only one $B$-harmonic function assuming the prescribed boundary values on the subset under consideration; or we can extend the class of $B$-harmonic functions in such a way that the extended class as far as possible still has the properties needed as tools for our investigations but, on the other hand, is so large that the boundary-value problem with functions prescribed on the whole distinguished boundary surface has always a solution.

While it seems that attempts in the first direction cannot possibly lead to successful results, it is quite natural in the case of a bicylinder to introduce as an extended class the class of functions which satisfy the system (1.1), denoted as doubly harmonic functions. On the other hand, it is clear that in the case of a domain different from a bicylinder the extended class must be defined by some other properties because the doubly harmonic functions do not have in general the required properties $\left({ }^{4}\right)$.

In the present paper, an attempt is made to define functions of extended class for a much larger family of domains with distinguished boundary surface than the family of bicylinders.

In introducing this extended class, we make the following requirements:

(1) In the case of a bicylinder, the extended class coincides with doubly harmonic functions.

(2) In the case of a domain which by a pseudo-conformal transformation can be obtained from a bicylinder, the class coincides with the functions obtained by a pseudo-conformal transformation from doubly harmonic functions.

(3) In the case where there exists a $B$-harmonic function which assumes prescribed values on the distinguished boundary surface, the function of the extended class coincides with the corresponding $B$-harmonic function.

(4) If to a function given on the distinguished boundary surface there exists a $B$-harmonic function which at every point of the distinguished boundary surface is larger (smaller) than the prescribed function $F$, then inside the domain $M^{4}$ this $B$-harmonic function is larger (smaller) than the function of the extended class corresponding to $F$.

Further, it is important for various purposes that our definition of functions of the extended class enable us to obtain a comparatively simple integral formula, representing functions of the extended class in $M^{4}$ in terms of their values on the distinguished boundary surface.

Roughly speaking, the basic idea of defining functions of extended class is as follows: Let us suppose that the real function $F$ is given on the distinguished boundary surface of a domain $M^{4}$. Now, we consider the totality

(4) We note that in general by a pseudo conformal mapping a doubly harmonic function is not transformed into a doubly harmonic function. 
$V\left(F ; M^{4}\right)$ of all $B$-harmonic functions which are regular in the domain $M^{4}$, continuous in the (closed) domain $\bar{M}^{4}$, and at every point of the distinguished boundary surface $M^{2}$ are equal to or larger than $F$. The totality of these functions we denote as the upper class $U\left(F ; M^{4}\right)$ of functions belonging to $F$. They form a normal family and at every interior point of $M^{4}$ the family has a minimum. These minimum values define a real function corresponding to $F$. This function can be defined by its boundary values on the whole boundary $m^{3}$ of $M^{4}$. Let us denote this function by $u(Z ; F)$.

In an analogous way, we consider the totality $\mathcal{L}\left(F ; M^{4}\right)$ of $B$-harmonic functions which are regular in $M^{4}$, continuous in $\bar{M}^{4}$, and at every point of $M^{2}$ equal to or smaller than $F$. As before, we show that the family has at every point a maximum $l(Z, F)$ and that this function possesses a boundary value on the boundary $m^{3}$. In general $u(Z ; F)$ does not equal $l(Z ; F)$ except in the case where there exists a $B$-harmonic function which assumes the values $F$ on $M^{2}$. On the other hand, as we shall show, on the boundary $m^{3}, u(Z ; F)=l(Z ; F)$ and therefore by our procedure we define on the boundary $m^{3}$ a function which we can associate with $F$, which we denote as the function of extended class, $E\left[Z ; F ; M^{4}\right], Z \in m^{3}$.

It remains now to define the functions of extended class inside the domain $M^{4}$. There exist several possibilities how to proceed. The most natural ways seem to be the two following ones:

I. Define as function of extended class( ${ }^{(5)} E h\left[Z ; F ; M^{4}\right]$ that harmonic function of four real variables which assumes on $m^{3}$ the values $u(Z ; F)=l(Z ; F)$ determined previously on $m^{8}$.

The functions $E h\left[Z ; F, M^{4}\right]$ possess the advantage that they are defined intrinsically, that is, they are independent of the choice of the coordinate system. On the other hand, they have the disadvantage that in the formula representing the function $E h\left[Z ; F, M^{4}\right]$ in $M^{4}$ in terms of the values $F$, there appears a Green's function of a four-dimensional domain $M^{4}$, and that we get a fourfold integration. Since the Green's function of four-dimensional domains are not sufficiently investigated and since operating with fourfold. integrals is often very inconvenient, this situation suggests replacing the above-mentioned class by another class of functions which on the boundary coincide with the values determined previously, which have a simpler representation in $M^{4}$, but which are not necessarily defined intrinsically.

II. Let $z_{k}=n_{k}\left(\mathrm{Z}, \mu_{1}, \mu_{2}\right), \kappa=1,2, \mu_{1}, \mu_{2}$ real, $\mathrm{Z}$ complex, be a two-dimensional family $\mathcal{F}$ of analytic surfaces such that to every point $Z^{(0)} \in \bar{M}^{4}$ there exists one and only one pair $\left(\mu_{1}, \mu_{2}\right)$ such that $z_{\kappa}=n_{\kappa}\left(Z, \mu_{1}, \mu_{2}\right), \kappa=1,2$, passes through $Z^{(0)}$ and $\mathcal{F}$ possesses some properties indicated in $\$ 4$.

We define as extended class $E n\left[Z ; F ; M^{4}\right]$ the totality of functions each of which is harmonic as function of $\operatorname{Re} \mathrm{Z}, \operatorname{Im} \mathrm{Z}$, in every $M^{4} \cap\left[z_{\kappa}=n_{\kappa}\left(\mathrm{Z}, \mu_{1}, \mu_{2}\right)\right.$,

(b) In general, we shall denote functions of the extended class by $E[$ ]. If we wish to stress that we consider those indicated under I or II, we shall write $E h[$ ] and $E n[$ ] respectively. (Thus if we write $E[$ ], the symbol refers to both types.) 
$\kappa=1,2]$ and assumes on $m^{3} \cap\left[z_{\kappa}=n_{\kappa}\left(\mathrm{Z}, \mu_{1}, \mu_{2}\right), \kappa=1,2\right]$ the values defined above.

Notation. The superscript attached to a symbol denoting a manifold indicates the dimension of the manifold.

In operating with sets we use the usual symbols: $\mathcal{S},+$ (sum set), - (difference set), $\cap$ (intersection), $\times$ (outer product of domains), and so on $\left(^{(6)}\right.$. For instance, we denoted by $\delta_{\lambda_{k} \in s_{k \alpha}^{1}} I_{k}^{2}\left(\lambda_{k}\right)$ the sum of a family of sets which depend upon a parameter $\lambda_{k}$ running through the interval $s_{k}^{1}$. (Note that the sets $I_{k}^{2}\left(\lambda_{k}\right)$ considered in this paper are families of disjoint segments of surfaces, lying in the four-dimensional space $\left[I_{k}^{2}\left(\lambda_{k}\right) \cap I_{k}^{2}\left(\lambda_{k}^{\prime}\right)=0\right.$, for $\left.\lambda_{k} \neq \lambda_{k}^{\prime}\right]$ so that $S_{\lambda_{k} \in s_{k \alpha}^{2}} I_{k}^{2}\left(\lambda_{k}\right)$ (under certain continuity conditions) is a segment of a hypersurface, that is, it is a three-dimensional manifold.

We denote by $M^{4}\left(z_{k}=z_{k}^{(0)}\right)$ the intersection of the domain $M^{4}$ with the plane $z_{k}=z_{k}^{(0)}$. We note that in the following we omit the superscript, and write simply $M^{4}\left(z_{\kappa}=z_{\kappa}\right)$.

We denote by $E[\cdots]$ the set of points whose coordinates satisfy the relations indicated in the brackets.

A point of the four-dimensional (Euclidian) space, with cartesian coordinates $x_{1}, y_{1}, x_{2}, y_{2}$ (or complex coordinates $z_{1}, z_{2}, z_{k}=x_{k}+i y_{k}$ ) is denoted by $Z$. In general we shall write $Z$ as argument rather than $\left(x_{1}, y_{1}, x_{2}, y_{2}\right)$ or $\left(z_{1}, z_{2}\right)$. When dealing with analytic functions of two complex variables, we shall use, however, as arguments $\left(z_{1}, z_{2}\right)$, in order to stress that a quantity is an analytic function of two complex variables.

We wish finally to remind the reader that in the usual manner we denote functions $U$ of four real variables $x_{1}, y_{1}, x_{2}, y_{2}$ which satisfy the equation $\partial^{2} U / \partial x_{1}^{2}+\partial^{2} U / \partial y_{1}^{2}+\partial^{2} U / \partial x_{2}^{2}+\partial^{2} U / \partial y_{2}^{2}=0$ as harmonic functions.

Functions $U$ of the same four real variables satisfying the system (1.1) will be denoted as doubly harmonic functions.

Finally, functions satisfying simultaneously systems (1.1) and (1.2) will be denoted as $B$-harmonic functions.

$B$-harmonic functions are real (or imaginary) parts of analytic functions of two complex variables $z_{1}, z_{2}, z_{k}=x_{k}+i y_{k}, \kappa=1,2$.

These functions are usually denoted as biharmonic functions. It seems preferable, however, to change this terminology since functions that satisfy the equation

$$
\frac{\partial^{4} U}{\partial x^{4}}+2 \frac{\partial^{4} U}{\partial x^{2} \partial y^{2}}+\frac{\partial^{4} U}{\partial y^{4}}=0
$$

are also denoted as biharmonic functions.

REMARK 1. Let us note that naturally one can obtain a generalized Cauchy formula representing the values of an analytic function of two variables inside the domain $\mathrm{H}^{4}$ in terms of its values on the whole three-dimensional

(8) See F. Hausdorff, Lehrbuch der Mengenlehre, 2d ed., 1927, p. 1. 
boundary $h^{3}$. We have

$$
\begin{aligned}
f\left(z_{1}, z_{2}\right)= & \frac{1}{4 \pi} \iiint_{h^{3}}\left\{f\left(\zeta_{1}, \zeta_{2}\right) \frac{\partial\left(1 / r^{2}\right)}{\partial \xi_{1}} d \eta_{1} d \xi_{2} d \eta_{2}-i \frac{\partial\left(1 / r^{2}\right)}{\partial \zeta_{1}} d \xi_{1} d \xi_{2} d \eta_{2}\right. \\
& \left.+\frac{\partial\left(1 / r^{2}\right)}{\partial \bar{\zeta}_{2}} d \xi_{1} d \eta_{1} d \eta_{2}-i \frac{\partial\left(1 / r^{2}\right)}{\partial \zeta_{2}} d \xi_{1} d \eta_{1} d \xi_{2}\right\}
\end{aligned}
$$

$\zeta_{k}=\xi_{k}+i \eta_{k}, \xi_{k}=\xi_{k}-i \eta_{k}$. See Bergman [7, p. 389](7). On the other hand, it is of importance that in the case of a large class of special domains there exists a generalized Cauchy formula expressing the values inside the domain in terms of values on a certain two-dimensional surface (distinguished boundary surface) of the domain.

REMARK 2. Every continuous function $u\left(\phi_{1} ; \phi_{2}\right)$ of two variables $\phi_{1}, \phi_{2}$ can be formally represented in the form of a double series,

$$
\begin{aligned}
\sum_{m=0}^{\infty} \sum_{n=-\infty}^{\infty}\left[A_{m n}^{(1)} \cos \left(m \phi_{1}+n \phi_{2}\right)+A_{m n}^{(2)} \sin \left(m \phi_{1}\right.\right. & \left.\left.+n \phi_{2}\right)\right] \\
& =\operatorname{Re}\left[\sum_{m=0}^{\infty} \sum_{n=-\infty}^{\infty} A_{m n} e^{i\left(m \phi_{1}+n \phi_{2}\right)}\right] .
\end{aligned}
$$

The above expression will represent the real part of an analytic function of two complex variables $\sum_{m=0}^{\infty} \sum_{n=0}^{\infty} A_{m n} z_{1}^{m} z_{2}^{n}$, if and only if $A_{m n}=0$, for $m=0,1,2, \cdots ; n=-1,-2$, that is, if $u\left(\phi_{1}, \phi_{2}\right)$ satisfies the conditions

$$
\int_{0}^{2 \pi} \int_{0}^{2 \pi} u\left(\phi_{1}, \phi_{2}\right) e^{i\left(m \phi_{1}-n \phi_{2}\right)} d \phi_{1} d \phi_{2}=0, m=1,2,3, \cdots ; n=1,2,3, \cdots \text {. }
$$

2. Domains $M^{4}$ and their structure. Before investigating functions of two complex variables in a class of domains with distinguished boundary surface, it is necessary to describe in a more exact manner the geometric structure of the domains under consideration. These domains have already been investigated in a series of previous papers $[1-11]\left({ }^{8}\right)$ in connection with other questions. In order to simplify the reading, we shall repeat briefly here the hypotheses needed concerning the geometrical structure of these domains and indicate the properties which will be needed for our purposes.

A. Segments of analytic hypersurfaces, which constitute the boundary of $M^{4}$. The boundary of the domains to be considered consists of finitely many segments of analytic hypersurfaces $\left({ }^{9}\right)$, denoted in the following by $i_{k}^{3}$,

(7) Numbers in brackets refer to the bibliography at the end of the paper.

(8) A knowledge of the previous papers indicated in the bibliography is not presupposed in the present paper.

(9) We note that of ten in the following for the sake of brevity we use the terms "hypersurface" and "surface" instead of the more precise terms "segment of a hypersurface" and "segment of a surface, $"$ respectively. 
$k=1,2, \cdots, n$. For the purpose of defining with the help of these hypersurfaces a domain with distinguished boundary surface, it is useful to extend each such hypersurface to a larger hypersurface $j_{\boldsymbol{k}}^{3}$ which divides a given domain $R^{4}$ into several parts. (See Fig. 1, p. 531). In general, the boundary of $j_{k}^{3}$ will belong to the boundary of $R^{4}$. For our purposes, it is only necessary that this extension, $j_{k}^{3}-i_{k}^{3}$ of $i_{k}^{3}$, be continuously differentiable. (Such an extension of a segment of an analytic hypersurface is in general possible, while the analytic extension is often impossible.) Thus, we shall work initially with $i_{k}^{3}$, returning later to $i_{k}^{3}$.

Suppose that for each $\lambda_{k} \in f^{1}=\mathrm{E}\left[0 \leqq \lambda_{k} \leqq 2 \pi\right]$

$$
\phi_{k}\left(Z, \lambda_{k}\right) \text {, }
$$

$Z \in R^{4}$, is a complex, continuously differentiable function of the five real variables $x_{1}, y_{1}, x_{2}, y_{2}, \lambda_{k}\left({ }^{10}\right)$. Let

$$
Q_{k}^{(2)}\left(\lambda_{k}\right)=\mathrm{E}\left[\phi_{k}\left(Z, \lambda_{k}\right)=0\right], \quad \lambda_{k} \in f^{1}=\mathrm{E}\left[0 \leqq \lambda_{k} \leqq 2 \pi\right]
$$

be a one-parameter family of surfaces or segments of surfaces in $R^{4}$. We suppose that $Q_{k}^{(2)}\left(\lambda_{k}\right)$ and $Q_{k}^{2}\left(\lambda_{k}^{\prime}\right)$ do not possess common points unless $\lambda_{k}=\lambda_{k}^{\prime}$, except perhaps when $\lambda=0$ and $\lambda^{\prime}=2 \pi$. In this latter case we assume that either $Q_{k}^{2}(0)=Q_{k}^{2}(2 \pi)$ or $Q_{k}^{2}(0)$ and $Q_{k}^{2}(2 \pi)$ have no common points.

We assume (Hypothesis 2.1) that these hypersurfaces can be uniformized, that is, there exist two functions of three real variables( $\left.{ }^{11}\right), \xi, \eta, \lambda_{k}$,

$$
h_{k}^{*}\left(\mathrm{Z}_{k}, \lambda_{k}\right), \quad \mathrm{Z}_{k} \in E_{k}^{2}\left(\lambda_{k}\right), \quad \mathrm{Z}_{k}=\xi+i \eta, \quad \lambda_{k} \in f^{1}, \quad \kappa=1,2,
$$

having continuous first derivatives with respect to $\xi, \eta$ and $\lambda_{k}, E_{k}^{2}\left(\lambda_{k}\right)$ being an appropriate domain in the $(\xi \eta)$-plane such that

$$
\phi_{k}\left[h_{k}^{1}\left(\mathrm{Z}_{k}, \lambda_{k}\right), h_{k}^{2}\left(\mathrm{Z}_{k}, \lambda_{k}\right), \lambda_{k}\right] \equiv 0,
$$

and such that to every point $Z^{(0)} \in R^{4^{\prime}}$ for which

$$
\phi_{k}\left(Z^{(0)}, \lambda_{k}^{(0)}\right)=0
$$

there is one and only one point $\left(\mathbf{Z}_{\mathbf{k}}^{0}, \lambda_{\mathbf{k}}^{0}\right)$ for which

$$
z_{k}^{0}=h_{k}^{k}\left(Z_{k}^{0}, \lambda_{k}^{0}\right) \text {, }
$$

$$
\kappa=1,2 .
$$

We shall further assume (2.2) $\left.{ }^{12}\right)$ that the boundary of $E_{k}^{2}\left(\lambda_{k}\right)$ is a Jordan curve and varies continuously (in the Fréchet sense) when $\lambda_{k}$ varies. It is to be

${ }^{(10)}$ It should be remarked that by an oversight in [6] we implied the existence of the derivatives with respect to $z_{1}$ and $z_{2}$. The present formulation is the correct one.

(11) In [1] the existence of derivatives with respect to $\mathrm{Z}_{k}$ was implied. It is correct as stated here.

(12) The term hypothesis will be omitted henceforth. 
noted that the domain [of the three-dimensional $\left(\xi \eta \lambda_{k}\right)$-space] defined by $S_{\lambda_{k} \in f^{1}} E_{k}^{2}\left(\lambda_{k}\right)$ is a cylinder (or a torus) (denoted by $d_{k}^{3}$ ) with varying crosssection, $E_{k}^{2}\left(\lambda_{k}\right)$. If $\left(\mathrm{Z}_{k}, \lambda_{k}\right)$ runs through all the points of $d_{k}^{3}$ then the set of points $\left(z_{1}, z_{2}\right), z_{k}=h_{k}^{k}\left(Z_{k}, \lambda_{k}\right)$, forms a hypersurface or a segment of a hypersurface denoted by $j_{k}^{3}$. $j_{k}^{3}$ is the sum of the laminas $Q_{k}^{2}\left(\lambda_{k}\right)$. By the correspondence

$$
\mathrm{Z}_{k} \rightarrow\left(z_{1}, z_{2}\right), \quad z_{k}=h_{k}^{*}\left(\mathrm{Z}_{k}, \lambda_{k}\right), \quad \mathrm{Z}_{k} \in E_{k}^{2}\left(\lambda_{k}\right),
$$

which we denote $\left.{ }^{13}\right)$ by $R_{\lambda_{k}}$, we have a one-to-one mapping of $E_{k}^{2}\left(\lambda_{k}\right)$ into $Q^{2}\left(\lambda_{k}\right)$. Using this notation we have, for example,

$$
E_{k}^{2}\left(\lambda_{k}\right)=R_{\lambda_{k}}^{-1}\left[Q_{k}^{2}\left(\lambda_{k}\right)\right]
$$

It is, for our purposes, necessary to know that $j_{k}^{3}$ has the property of dividing every sufficiently small hypersphere $N_{k}^{4}$ with center at a point $Z^{(0)}$ of $j_{k}^{3}$ into two connected parts. To this end, we assume (2.3) that at every point $Z$ of $j_{k}^{3}$ at least one of the determinants

$$
\frac{\partial\left(\phi_{k}^{(1)}, \phi_{k}^{(2)}\right)}{\partial\left(\lambda_{k}, X\right)} \neq 0, \quad \phi_{k}=\phi_{k}^{(1)}+i \phi_{k}^{(2)}
$$

here $X$ represents any one of the four variables $x_{1}, y_{1}, x_{2}, y_{2}$, and

$$
0<\left|\frac{\partial \phi_{k}}{\partial \lambda_{k}}\right|<\infty \text {. }
$$

Indeed suppose $\partial \phi_{k}^{(1)} / \partial \lambda_{k} \neq 0$. Then from $\phi_{k}^{(1)}\left(Z, \lambda_{k}\right)=0$, it follows that $\lambda_{k}=\lambda_{k}(Z)$. Substituting the last expression into $\phi_{k}^{(2)}\left(Z, \lambda_{k}\right)$ we obtain the equation of (2.2) in the form $\phi_{k}^{(2)}\left[Z, \lambda_{k}(Z)\right]=0$. Since

$$
\frac{d \phi_{k}^{(2)}\left[Z, \lambda_{k}(Z)\right]}{d x_{1}}=\frac{\partial \phi_{k}^{(2)}}{\partial x_{1}}+\frac{\partial \phi_{k}^{(2)}}{\partial \lambda_{k}} \frac{\partial \lambda_{k}}{\partial x_{1}}, \quad \frac{\partial \phi_{k}^{(2)}}{\partial x_{1}} \equiv\left[\frac{\partial \phi_{k}^{(2)}\left[Z, \lambda_{k}\right]}{\partial x_{1}}\right], \quad \lambda_{k}=\lambda_{k}(Z),
$$

and from $\phi_{k}^{(1)}\left[Z, \lambda_{k}(Z)\right] \equiv 0$, we have

$$
\frac{\partial \lambda_{k}}{\partial x_{1}}=-\frac{\partial \phi_{k}^{(1)}}{\partial x_{1}} / \frac{\partial \phi_{k}^{(1)}}{\partial \lambda_{k}}
$$

we obtain finally

$$
\frac{d \phi_{k}^{(2)}}{d x_{1}}=\frac{\partial\left[\phi_{k}^{(2)}, \phi_{k}^{(1)}\right]}{\partial\left[x_{1}, \lambda_{k}\right]} / \frac{\partial \phi_{k}^{(1)}}{\partial \lambda_{k}} .
$$

(18) We note that the mapping of one segment $E_{k}^{2}\left(\lambda_{k}\right)$ into the corresponding segment $Q_{k}^{(2)}\left(\lambda_{k}\right)$ of the $Z$-space is denoted by $R_{\lambda_{k}}$.

The mapping of the entire three-dimensional manifold $\bar{d}^{3}=S_{\lambda_{k} \in f^{1}} \overline{E_{k}^{2}}\left(\lambda_{k}\right)$ onto $S_{\lambda_{k} \in f^{1}} \bar{Q}_{k}^{(2)}\left(\lambda_{k}\right)$ will be denoted by $\boldsymbol{R}_{\boldsymbol{k}}$. 
It follows therefore from (2.9) and (2.10) that (2.2) can be written in the form $x_{1}=\kappa\left(y_{1}, x_{2}, y_{2}\right)$ which implies the property of $j_{k}^{3}$ mentioned above, see [1, pp. 79-81].

B. The domain $M^{4}$. Let us consider $n$ hypersurfaces $j_{k}^{3}, k=1,2,3, \cdots, n$, of the form described in A. We shall suppose that the $j_{k}^{3}, k=1,2, \cdots, n$, divide the domain $R^{4}$, now taken to be simply connected, into parts in such a

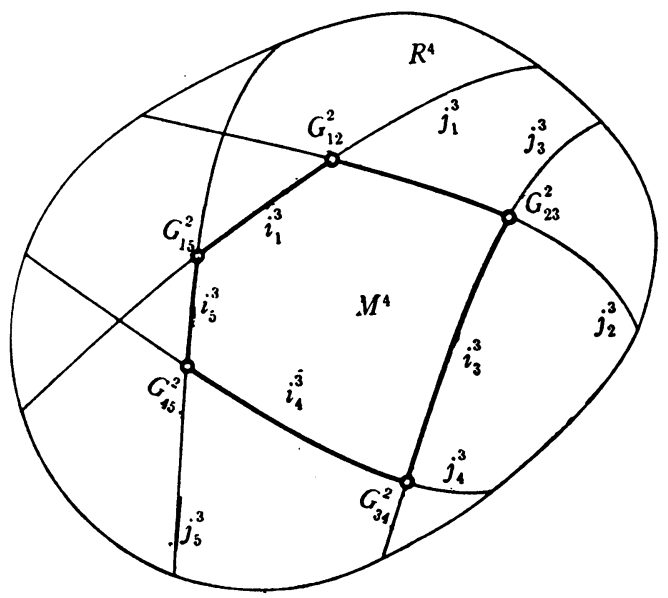

Fig. 1. Two-dimensional analogue of domains $R^{4}$ and $M^{4}$, of boundrry $m^{3}$ of $M^{4}$, and distinguished boundary surface ${ }^{(14)} M^{2}=S_{k=1}^{n} S_{e-1}^{\prime n} \bar{G}_{k s}^{2}$.

manner that there exists at least one connected domain $M^{4}$ (one of the cells into which $R^{4}$ is divided by the $\left.j_{k}^{3}, k=1, \cdots, n\right)$ which lies wholly in $R^{4}$ (for example, $\bar{M}^{4} \subset R^{4}$ ). The entire boundary $m^{3}$ of $M^{4}$ consists therefore of parts of $j_{k}^{3}$. We note that $M^{4}$ by definition has no points in common with any $j_{k}^{3}, k=1,2, \cdots, n$. We shall suppose (2.4) that the parts of $j_{k}^{3}$ belonging to the boundary $m^{3}$ of $M^{4}$,

$$
\bar{i}_{k}^{8}=j_{k}^{3} \cap \bar{M}^{4}
$$

where

$$
\bar{i}_{k}^{8}=\underset{\lambda_{k} \in \rho^{1}}{\mathcal{S}} I_{k}^{2}\left(\lambda_{k}\right), \quad I_{k}^{2}\left(\lambda_{k}\right)=Q_{k}^{2}\left(\lambda_{k}\right) \cap \bar{M}^{4},
$$

are segments of analytic hypersurfaces, that is, in a certain neighborhood of $I_{k}^{2}\left(\lambda_{k}\right), \phi_{k}\left(z_{1}, z_{2}, \lambda_{k}\right)$ (see (2.1)) is an analytic function of two complex variables $z_{1}, z_{2}$. Also the uniformizing functions $h_{k}^{\alpha}\left(Z_{k}, \lambda_{k}\right), \kappa=1,2, k=1,2, \cdots, n$

(14) Note that in the model the dimensions of the manifolds are diminished by 2, that is, hypersurfaces are replaced by lines, surfaces by points, and so on. 
(see (2.3)), are analytic functions of $\mathrm{Z}_{k}$ in the corresponding part $R_{\lambda_{k}}^{-1}\left[I_{k}^{2}\left(\lambda_{k}\right)\right]$ of $E_{k}^{2}\left(\lambda_{k}\right)$.

In order to avoid too great generality, we shall suppose (2.5) that $B_{k}^{2}\left(\lambda_{k}\right)$ $=R_{\lambda_{k}}^{-1}\left[I_{k}^{2}\left(\lambda_{k}\right)\right]$ and therefore $I_{k}^{2}\left(\lambda_{k}\right)$ is a simply-connected domain or possibly

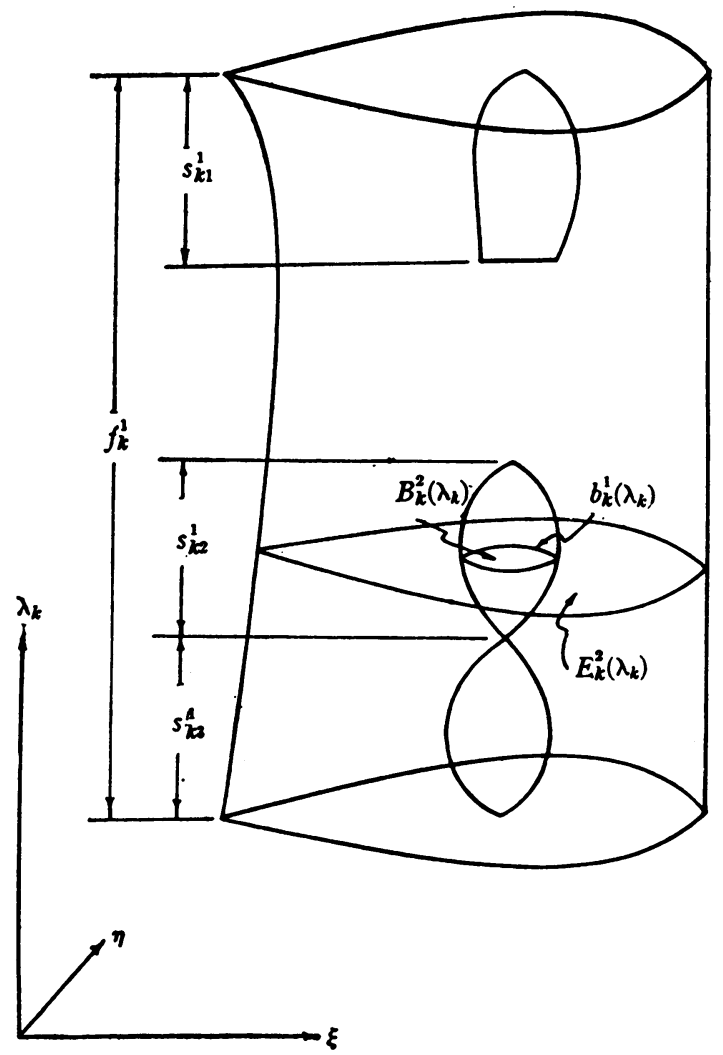

Fig. 2. Domain (15) $d^{3}$ (the image of $j^{3}$ in the $\xi \eta \lambda_{k}$ space), $\mathrm{Z}_{k}=\xi+i \eta$.

empty, for all $\lambda_{k} \in f^{1}$ except at most in a set $n^{0}$ consisting of a finite number of points. Further, we assume (2.6) that the set of values of the $\lambda_{k}$ 's belonging to $f^{1}-n^{0}$ for which the $I_{k}^{2}\left(\lambda_{k}\right)$ are not empty consists of finitely many connected segments $s_{k \alpha}^{1}, \alpha=1,2, \cdots, \beta$. For every $\lambda_{k}^{(0)} \in s_{k \alpha}^{1}, B_{k}^{2}\left(\lambda_{k}^{(0)}\right)$ represents the kernel domain in the sense of Carathéodory of $B_{k}^{2}\left(\lambda_{k}\right)$, when $\lambda_{k} \rightarrow \lambda_{k}^{(0)}$.

We assume. (2.7) that for $\lambda_{k} \in s_{k \alpha}^{1}$, the boundary $b_{k}^{1}\left(\lambda_{k}\right)$ of $B_{k}^{2}\left(\lambda_{k}\right)$ consists of finitely many, at most $\kappa$, differentiable arc segments, which meet each other

(15) $S_{\alpha=1}^{\beta_{k}} S_{\lambda_{k} \in s_{k \alpha}^{1}} B^{2}\left(\lambda_{k}\right)$ is the image of a segment $i_{k}^{8}$ of analytic hypersurface. $S_{k} i_{k}^{3}$ constitutes the boundary $m^{2}$ of $M^{4}$. 
under nonvanishing angles. $\kappa$ is assumed to be independent of $\lambda_{k}$. For $\lambda_{k} \in \bar{s}_{k \alpha}^{1}-s_{k \alpha}^{1}, B_{k}^{2}\left(\lambda_{k}\right)$ is assumed (2.8) either to consist of an isolated arc, which possibly may degenerate into isolated points, or of (two-dimensional) domains part of whose boundaries degenerate to isolated curves or points.

On each boundary curve $b_{k}^{1}\left(\lambda_{k}\right)$ of $B_{k}^{2}\left(\lambda_{k}\right)=R_{\lambda_{k}}^{-1}\left[I_{k}^{2}\left(\lambda_{k}\right)\right], \lambda_{k} \in s_{k \alpha}^{1}$, we may define a sense of direction such that it is positive when $R_{\lambda_{k}}^{-1}\left[I_{k}^{2}\left(\lambda_{k}\right)\right]$ lies to the left (2.9). If, approaching $\lambda_{k}^{(0)} \in \bar{s}_{k \alpha}^{1}-s_{k \alpha}^{1}$, from both sides, a component of the boundary $R_{\lambda_{k}}^{-1}\left[I_{k}^{2}\left(\lambda_{k}\right)\right]$ degenerates to an open arc or to a point, the sense of direction of the corresponding curves on $R_{\lambda_{k}}^{-1}\left[I_{k}^{2}\left(\lambda_{k}^{+}\right)\right]$and $R_{\lambda_{k}}^{-1}\left[I_{k}^{2}\left(\lambda_{k}^{-}\right)\right]$ should be the same.

C. The distinguished boundary surface. As indicated in the introduction, of special importance is the surface consisting of intersections of boundary hypersurfaces $\bar{i}_{\mathbf{k}}^{3}$ which we denote as the distinguished boundary surface. $\left(\bar{i}^{3}\right.$ denotes the closure of $i^{3}$.)

We assume (2.10) that every intersection $\bar{i}_{k}^{3} \cap \bar{i}_{\sigma}^{3}, \sigma \neq k ; k=1,2, \cdots, n$; $\sigma=1,2, \cdots, n$, is a two-dimensional manifold.

As has already been indicated in previous investigations on the boundary $m^{3}=S_{k-1}^{n} i_{k}^{3}$ of $M^{4}$, the following two types of points can be distinguished:

I. $J$-points that belong to only one hypersurface $i_{\mathbf{k}}^{3}$.

II. $K$-points that belong to the intersection of at least two $\bar{i}_{\mathbf{k}}^{3}$. By considerations similar to those of $[2$, p. 83$]$, we can show that every point of $\boldsymbol{R}_{\boldsymbol{\lambda}_{k}}\left[b_{\boldsymbol{k}}^{1}\left(\lambda_{k}\right)\right]$ must be a $K$-point.

$\boldsymbol{R}_{\lambda_{k}}\left[b_{k}^{1}\left(\lambda_{k}\right)\right]$ (the image of $b_{k}^{1}\left(\lambda_{k}\right)$ in the $Z$-space) will be denoted by $i_{k}^{1}\left(\lambda_{k}\right)$. Since every point of $i_{k}^{1}\left(\lambda_{k}\right)$ is a $K$-point, that is, is an intersection of at least two $i_{m}^{3}$, it must belong to at least one $i_{\sigma}^{3}, \sigma \neq k$. We write

$$
\begin{array}{cc}
\bar{i}_{k \sigma}^{1}\left(\lambda_{k}\right)=\bar{i}_{k}^{1}\left(\lambda_{k}\right) \cap \bar{i}_{\sigma}^{3}, & \sigma \neq k, \\
\bar{G}_{k \sigma}^{2}=\int_{\lambda_{k} \in \bar{j}_{k \alpha}^{1}} i_{k \sigma}^{1}\left(\lambda_{k}\right), \quad G_{k}^{2}=\int_{\sigma=1}^{n} \bar{G}_{k \sigma}^{2} . &
\end{array}
$$

We note that naturally $\left.{ }^{16}\right) i_{k}^{1}\left(\lambda_{k}\right)=S_{\sigma=1}^{\prime n} i_{k \sigma}^{1}\left(\lambda_{k}\right)$ and that the intersection of two $\bar{G}_{k \sigma}^{2}$ is not necessarily empty, since a $K$-point can belong to more than two $\bar{i}_{m}^{3}$ 's.

$$
M^{2}=\oint_{k=1}^{n} \bar{G}_{k}^{2}=\oint_{k=1}^{n}{\underset{\delta}{\sigma=1}}_{S^{\prime}}^{n} \bar{G}_{k \sigma}^{2}
$$

represents the distinguished boundary surface of $\left({ }^{17}\right) M^{4}$.

(16) The prime attached to $S$ indicates that the term $\sigma=k$ is omitted.

${ }^{17}$ We wish to note that in previous papers we formulated some additional hypotheses and proved some further properties of the $G_{k}^{2 s}$ s, for example, the possibility of carrying out an integration of functions defined on $M^{2}$. Since, however, for the purposes of the present paper these properties are not needed, we omit a discussion of these questions.' 
3. Further assumptions concerning the structure of $M^{4}$. In the present paper we consider a special class of domains, namely, those bounded by finitely many analytic hypersurfaces. The general geometric structure of these domains has been described in $\$ 2$. In order, however, to be able to prove certain properties of functions of extended class, we have to make additional special hypotheses, to be formulated in the present section. These hypotheses will insure that to every lamina, say $\left({ }^{18}\right) I^{2}=I_{k}^{2}\left(\lambda_{k}\right)$, it is possible to construct a domain $A^{4}$ of the following type.

Let $T^{4}=I^{2} \times R^{2}$ be a product domain, one of whose components is $I^{2}$, and the second one a suitably chosen domain $R^{2}$, see (3.8). By a suitable pseudoconformal transformation, we obtained from $T^{4}$ a domain $A^{4}$ to whose boundary the lamina $I^{2}=I_{k}^{2}\left(\lambda_{k}\right)$ belongs and which domain includes $M^{4}$. A domain $A^{4}$ of this type is denoted as an "exterior domain of comparison with respect to the lamina $I_{k}^{2}\left(\lambda_{k}\right)$."

We proceed now to the formulation of the hypotheses which insure the existence of the domain of comparison $A^{4}$ described above $\left({ }^{19}\right)$.

According to (2.4) the segment $I^{2}=I_{k}^{2}\left(\lambda_{k}\right)$ of an analytic hypersurface belonging to the boundary $m^{3}$ can be given in the parametric form

$$
z_{k}=h_{k}^{\kappa}\left(\mathbf{Z}_{k}, \lambda_{k}\right) \text {, }
$$$$
\kappa=1,2 ; \lambda_{k} \text { fixed, }
$$

where $h_{\mathbf{k}}^{k}$ are continuously differentiable functions of the complex variable $\mathbf{Z}_{k}$ and a real variable $\lambda_{k}$ defined for $\mathrm{Z}_{k} \in B_{k}^{2}\left(\lambda_{k}\right), \quad \lambda_{k} \in s_{k \alpha \alpha}^{1}, \quad s_{k \alpha}^{1}=E\left[\lambda_{k}^{(\alpha)}\right)<\lambda_{k}$ $\left.<\lambda_{k}^{(\alpha+1)}\right]$. These functions for every fixed value of $\lambda_{k} \in s_{k \alpha}^{1}$ are analytic functions of the complex variable $\mathbf{Z}_{k}$. For every fixed value $\lambda_{k}, \mathbf{Z}_{k}$ can be eliminated from (3.1) and we can write the equation of the surface in which $I_{k}^{2}\left(\lambda_{k}\right)$ lies in the form

$$
\phi_{k}\left(z_{1}, z_{2}, \lambda_{k}\right)=0 \text {. }
$$

We assume $\left(2.4^{\prime}\right)\left({ }^{20}\right)$ that $\phi_{k}\left(z_{1}, z_{2}, \lambda_{k}\right)$ is an analytic function of two complex variables $z_{1}, z_{2}$ in $\bar{M}^{4}$ and that (3.1) there exists another continuously differentiable function, say $g_{k}\left(z_{1}, z_{2}, \lambda_{k}\right)$, which is, for every $z_{1}, z_{2}$ belonging to $\bar{M}^{4}$, an analytic function of these two variables possessing the property that the pseudo-conformal transformation

$$
z_{1}^{*}=\phi_{k}\left(z_{1}, z_{2}, \lambda_{k}\right), \quad z_{2}^{*}=g_{k}\left(z_{1}, z_{2}, \lambda_{k}\right)
$$

(18) We required that for every lamina $I_{k}^{2}\left(\lambda_{k}\right), \lambda_{k} \in s_{k \alpha}^{1}, k=1,2, \cdots, n, \alpha=1,2, \cdots, \beta_{k}$, the conditions described here for one lamina $I^{2}$ are fulfilled.

(19) Exterior domains of comparison of this type have been extensively studied in [8]. They were used in order to prove that the kernel function becomes infinite of certain order upon approach to the boundary, as well as to investigate the behavior of analytic functions of two complex variables in the neighborhood of laminas of this kind.

${ }^{(20)}\left(2.4^{\prime}\right)$ is a reinforgement of (2.4) according to which function $\phi_{k}\left(z_{1}, z_{2}, \lambda_{k}\right)$ was assumed to be an analytic function of $z_{1}, z_{2}$ only in a certain neighborhood $N^{4}$ of $\phi_{k}\left(z_{1}, z_{2}, \lambda_{k}\right)=0$. 
is one-to-one and schlicht in $\bar{M}^{4}$. The domain obtained from $\bar{M}^{4}$ by the transformation (3.3) will be denoted by $\bar{M}^{* 4}$.

The new coordinates $z_{1}^{*}, z_{2}^{*}$ will be denoted as "canonical coordinates with respect to the lamina $I_{k}^{2}\left(\lambda_{k}\right)$." We assume also that the origin, that is, $z_{2}^{*}=0$, is an interior point of the domain $I_{k}^{* 2}\left(\lambda_{k}\right)$ (situated in the plane $z_{1}^{*}=0$ ), so that the origin lies inside the segment $I_{k}^{* 2}\left(\lambda_{k}\right)$. (This can always be realized by adding to $g_{k}$ a suitably chosen constant.)

(3.2) The lamina (21) $I_{k}^{* 2}\left(\lambda_{k}\right)$ which represents a domain of the $z_{2}^{*}$-plane (denoted by $I^{* 2}$ ), which domain lies in the plane $z_{1}^{*}=0$, is assumed to be a star domain with the center at the origin and to include in its interior a circle $\mathrm{E}\left[\left|z_{2}^{*}\right| \leqq \rho\right]$.

(3.3) $M^{* 4}$ is a bounded domain. Without any loss of generality we may assume (3.3a): For $\left(z_{1}^{*}, z_{2}^{*}\right)$ belonging to $M^{* 4}$, we have

$$
\left|z_{1}^{*}\right|<1 / 2, \quad\left|z_{2}^{*}\right|<1 .
$$

(3.4) The segment

$$
\mathrm{E}\left[0>x_{1}^{*}>-1, y_{1}^{*}=0\right]
$$

of the hyperplane $y_{1}^{*}=0$ does not belong to $M^{* 4}$.

(3.5) There exist an $r_{1}^{(0)}, r_{1}^{(0)}>0$, a $\mu^{(0)}, 0<\mu^{(0)}<\infty$, and a $\sigma_{1}, \sigma_{1}<1 / 2$ such that for ${ }^{(22)}\left|z_{1}^{*}\right|<r_{1}^{(0)}$

$$
M^{* 4}\left(z_{1}^{*}=z_{1}^{*}\right) \subset I^{* 2} /\left(1-\sigma_{1}\left|z_{1}^{*}\right|^{1 / \mu^{(0)}}\right), \quad I^{* 2} \equiv \bar{M}^{* 4}\left(z_{1}^{*}=0\right)
$$

holds.

(3.6) There exists a $\mu^{(1)}, \mu^{(1)}<\infty$, such that $\left.{ }^{23}\right)$

$$
\frac{I^{* 2}}{1-s / 2} \subset \frac{I^{* 2}}{1-s} \exp \left[i s / \nu_{1}\right] \text { for } \nu_{1} \geqq \mu^{(1)}, \exp [a] \equiv e^{a} \text {. }
$$

THEOREM 3.1. Let ${ }^{(24)} A^{4}$ denote the domain of the $z_{1}^{*}, z_{2}^{*}$-space obtained from

$$
T^{4}=I^{* 2} \times R^{2}, R^{2}=\mathrm{E}\left[\left|z_{1}^{*}\right|<1 / 2\right]-\mathrm{E}\left[-1 / 2<x_{1}^{*}<0, y_{1}^{*}=0\right]
$$

by a transformation

$$
z_{1}^{* *}=z_{1}^{*}, \quad z_{2}^{* *}=z_{2}^{*} /\left(1-\sigma z_{1}^{* 1 / \mu}\right),
$$

see Fig. 3.

(21) $I_{k}^{* 2}\left(\lambda_{k}\right)=M^{* 4}\left(z_{1}^{*}=0\right)$ is the image of $I_{k}^{2}\left(\lambda_{k}\right)$ in the $z_{1}^{*}, z_{2}^{*}$-space.

(22) $M^{4}\left(z_{1}^{*}=z_{1}^{*}\right)$ is an abbreviation for the intersection of $M^{*}$ and the plane $z_{1}^{*}=z_{1}^{*(0)}$. Note that we omit the superscript (0).

(23) $I^{* 2} / a$ denotes the domain which we obtain from $I^{* 2}$ by the transformation $Z=z^{*} / a$. $I^{* 2} \exp (i b)$ means the domain obtained by the transformation $Z=z^{*} e^{i b}$.

(24) We note that the domain $A^{4}$ is located in the $z_{1}^{*}, z_{2}^{*}$-space. 
Suppose further that $\epsilon$ is some number such that $0<\epsilon<1 / 2$, and $\sigma$ is chosen in such a way that

$$
1>\sigma>2 \sigma_{1} /(1-\epsilon) \quad \text { and }
$$$$
\text { (3.10b) } \quad 1-\sigma<\rho
$$

(see (3.2)) hold.

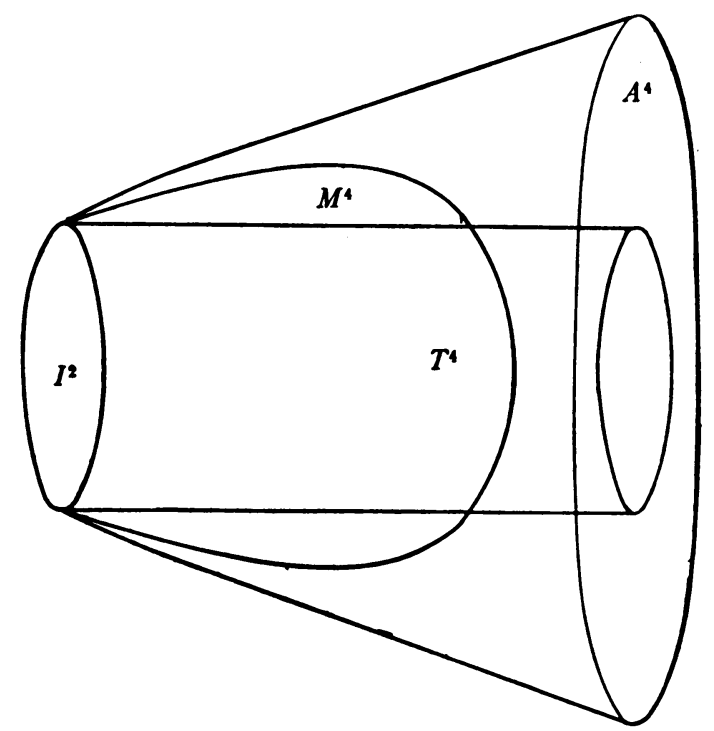

Fig. 3. Three-dimensional analogue of the domains(25) $M^{4}, T^{4}$ and $A^{4}$.

Finally, let $\mu$ be chosen so large that

$$
\begin{aligned}
\cos (\pi / \mu)-(\pi / \mu) & >1 / 2, \\
\cos (\pi / \mu)-\sin (\pi / \mu) & >1-\epsilon, \\
\gamma \equiv \mu(1-\sigma)(1-\epsilon) & >\pi \mu^{(1)}, \\
1-\sigma\left(r_{1}^{(0)}\right)^{1 / \mu} \cos (\pi / \mu)+\sigma(\pi / \mu) & <\rho, \\
\mu & >\mu^{(0)} .
\end{aligned}
$$

\section{Then}

$$
M^{* 4} \subset A^{4}
$$

REMARK. We note that it follows from our hypotheses that the constants $\sigma, \mu$ and $\rho$ can always be chosen in accordance with (3.10a)-(3.10e).

Proof. (1) We consider at first the intersections $A^{4}\left(z_{1}^{*}=z_{1}^{*}\right)$ for which $\left|z_{1}^{*}\right|^{\prime} \geqq r_{1}^{(0)} . I^{* 2}$ includes the circle $\left|z_{2}^{*}\right|<\rho$. For a fixed $z_{1}^{*}$ the transformation (3.9) means a dilatation of $I^{* 2}$ by the factor $1 /\left|1-\sigma z_{1}^{* 1 / \mu}\right|$ and a rotation.

${ }^{(25)}$ Note that the domain $R^{2}$ (see (3.8)) is replaced here by a segment of a straight line. 
We have

$$
\left|1-\sigma z_{1}^{* 1 / \mu}\right| \leqq\left[\left(1-\sigma r_{1}^{1 / \mu} \cos \left(\phi_{1} / \mu\right)\right)^{2}+\left(\sigma r_{1}^{1 / \mu} \sin \left(\phi_{1} / \mu\right)\right)^{2}\right]^{1 / 2}
$$

By (3.11d)

$$
\leqq 1-\sigma\left(r_{1}^{(0)}\right)^{1 / \mu} \cos (\pi / \mu)+\sigma(\pi / \mu), \quad z_{1}^{*}=r_{1} e^{i \phi_{1}}
$$

By $(3.11 d)$

$$
\frac{\rho}{1-\sigma\left(r_{1}^{(0)}\right)^{1 / \mu} \cos (\pi / \mu)+\sigma(\pi / \mu)}>1
$$

which means that the circle $E\left[\left|z_{2}^{*}\right|<\rho\right]$, see (3.2), is so expanded that it covers the unit circle. Therefore for $\left|z_{1}^{*}\right| \geqq r_{1}^{(0)}$

$$
A^{4}\left(z_{1}^{*}=z_{1}^{*}\right) \supset \mathrm{E}\left[\left|z_{2}^{*}\right|<1\right] \supset M^{* 4}\left(z_{1}^{*}=z_{1}^{*}\right)
$$

holds.

(2) We consider now intersections $A^{4}\left(z_{1}^{*}=z_{1}^{*}\right)$ for which

$$
\left|z_{1}^{*}\right|<r_{1}^{(0)}
$$

By definition

$$
A^{4}\left(z_{1}^{*}=z_{1}^{*}\right)=\frac{I^{* 2}}{\left|1-\sigma z_{1}^{* 1 / \mu}\right|} \exp (i \delta)
$$

where

$$
\delta=\arctan \left[\frac{\sigma r_{1}^{1 / \mu} \sin \left(\phi_{1} / \mu\right)}{1-\sigma r_{1}^{1 / \mu} \cos \left(\phi_{1} / \mu\right)}\right] .
$$

Now, by (3.18) and (3.11c)

$$
\begin{aligned}
|\delta| & \leqq \frac{\sigma r_{1}^{1 / \mu} \pi / \mu}{1-\sigma\left(r^{(0)}\right)^{1 / \mu}} \\
& =\sigma(1-\epsilon) r_{1}^{1 / \mu} \frac{\pi}{\mu\left(1-\sigma\left(r_{1}^{(0)}\right)^{1 / \mu}\right)(1-\epsilon)}=\frac{\pi \sigma(1-\epsilon) r_{1}^{1 / \mu}}{\nu}
\end{aligned}
$$

where from (3.7) follows that $\nu>\mu^{(1)}$.

Further, by (3.11b)

$$
\begin{aligned}
\left|1-\sigma z_{1}^{* 1 / \mu}\right| & \leqq 1-\sigma r_{1}^{1 / \mu} \cos (\pi / \mu)+\sigma r_{1}^{1 / \mu} \sin (\pi / \mu) \\
& =1-\sigma r_{1}^{1 / \mu}[\cos (\pi / \mu)-\sin (\pi / \mu)] \\
& \leqq 1-(1-\epsilon) \sigma r_{1}^{1 / \mu}
\end{aligned}
$$


holds. Therefore

$$
\begin{aligned}
A^{4}\left(z_{1}^{*}=z_{1}^{*}\right) & =\frac{I^{* 2}}{\left|1-\sigma z_{1}^{* 1 / \mu}\right|} \exp (i \delta) \\
& \supset \frac{I^{* 2}}{1-(1-\epsilon) \sigma r_{1}^{1 / \mu}} \exp \left(i \pi \frac{\sigma(1-\epsilon) r_{1}^{1 / \mu}}{\nu}\right), \quad \nu>\pi \mu^{(1)} .
\end{aligned}
$$

By (3.7), (3.10a), (3.11e) and (3.6) it follows that for $\left|z_{1}^{*}\right|<r_{1}^{(0)}$

$$
A^{4}\left(z_{1}^{*}=z_{1}^{*}\right) \supset \frac{I^{* 2}}{1-2^{-1}(1-\epsilon) \sigma r_{1}^{1 / \mu}} \supset \frac{I^{* 2}}{1-\sigma_{1} r_{1}^{1 / \mu(0)}} \supset M^{* 4}\left(z_{1}^{*}=z_{1}^{*}\right) \text {. }
$$

(3.15) and (3.22) together with (3.4) implies (3.12), that is, the statement of our theorem.

Finally, we make the hypothesis (3.8) needed in the following: There exist fixed constants $H, \tau^{\prime}, \rho^{\prime}$, such that to every point $\left(z_{1}^{*}, z_{2}^{*}\right)$ of $M^{* 2}$ for which $\left|z_{1}^{*}\right| \leqq \rho^{\prime}$, there exists a point $\left(0, z_{2}^{*(0)}\right)$ of $i_{\mathbf{k}}^{1}\left(\lambda_{k}\right)$ such that

$$
\left|z_{2}^{*}-z_{2}^{*(0)}\right| \leqq A\left|z_{1}^{*}\right|^{1 / \tau^{\prime}} \text {. }
$$

Remark. Applying the methods developed in [1] and using Theorem 3.1 it is possible to obtain bounds for the kernel function, see [1], when we approach to points of the distinguished boundary surface, $M^{2}$.

4. The two-parameter family of analytic surfaces $\left[z_{1}=Z, z_{2}=\mu_{1}+i \mu_{2}\right]$ and their properties. In the case of functions $E h\left[Z ; F ; M^{4}\right]$ of extended class, the additional hypothesis which has to be made on $M^{4}$ to insure the possibility of construction of functions $E h\left[Z ; F ; M^{4}\right]$ is: (4.1) Green's function for harmonic functions of four variables exists for the domain $M^{4}$. ((4.1) implies that the domain $M^{4}$ has the property that if on its boundary $m^{3}$ a continuous function is given, then at every point the corresponding harmonic function converges to the given function when we approach the boundary.

In the case of functions $E n\left[Z ; F ; M^{4}\right]$ the corresponding hypotheses be: come more complicated. For simplicity's sake, we shall formulate these hypotheses in the special case where

$$
z_{1}=n_{1} \equiv \mathrm{Z}, \quad z_{2}=n_{2} \equiv \mu_{1}+i \mu_{2} .
$$

We assume in this case

(4.1a) The projection $P^{2}$ of $M^{4}$ on the $z_{2}$-plane, that is, the totality of $z_{2}$ values of all points $\left(z_{1}, z_{2}\right)$ of $M^{4}$, is a two-dimensional domain of finite connectivity.

(4.2a) The boundary of $M^{4}\left(z_{2}=z_{2}\right)$ is a one-dimensional manifold which, except perhaps for a finite set of values $z_{2}$, consists of finitely many continuously differentiable curve segments, which form nonvanishing angles with each other. 
(4.3a) For every $z_{2} \in \bar{P}^{2}, M^{4}\left(z_{2}=z_{2}\right)$ can be written as a sum of finitely many (at most $s$ ) connected domains, $M^{4}\left(z_{2}=z_{2}\right)=\oint_{\alpha} N_{\alpha}^{2}\left(z_{2}\right)$.

We assume that for every $z_{2}^{(0)} \in \bar{P}^{2}$, the following alternatives take place: Either (1) the boundary of $N_{\alpha}^{2}\left(z_{2}\right)$ when $z_{2} \rightarrow z_{2}^{(0)}$ converges in the Fréchet sense to the boundary of $N_{\alpha}^{2}\left(z_{2}^{(0)}\right)$ and therefore $N_{\alpha}^{2}\left(z_{2}^{(0)}\right)$ is the kernel domain (in the sense of Carathéodory) of $N\left(z_{2}\right)$ when $z_{2} \rightarrow z_{2}^{(0)}$, or (2) the boundaries of $N_{\alpha}^{2}\left(z_{2}\right)$ for $z_{2} \rightarrow z_{2}^{(0)}$ converge to a point and therefore $\lim _{z_{2} \rightarrow z_{2}} N_{\alpha}^{(0)} N_{\alpha}^{2}\left(z_{2}\right)$ is empty, or finally (3) $\lim _{z_{2} \rightarrow z_{2}} N_{\alpha}^{2}\left(z_{2}\right)$ is a domain, say $\tilde{N}_{\alpha}^{2}$, but $\tilde{N}_{\alpha}^{2}$ does not belong to $M^{4}\left(z_{2}=z_{2}^{(0)}\right)$. Then $\tilde{N}_{\alpha}^{2} \in \bar{M}^{4}\left(z_{2}=z_{2}^{(0)}\right)$ and it consists of $J$-points, the boundary (which consists of $K$-points) of $\tilde{N}_{\alpha}^{2}$ satisfies hypothesis 4.2a and $\tilde{N}_{\alpha}^{2}$ is the kernel of $N_{\alpha}^{2}\left(z_{2}\right)$, when $z_{2} \rightarrow z_{2}^{(0)}$.

5. Some properties in $m^{3}$ of the functions of extended class. In $\S \S 2$ and 3 we formulated certain hypotheses which have to be made about the structure of the domain $M^{4}$ and its boundary. In this and in the next section, we shall show that these hypotheses insure that certain properties of a real function $F(Z)$ defined on $M^{2}$ imply certain properties of the function $E\left(Z ; M^{4} ; F\right)$ in $m^{3}$. We shall prove, namely, two theorems.

THEOREM 5.1. If $F(Z)$ is a (uniformly) continuous function in $M^{2}$, then in every $i_{k}^{3}$ the function $E\left(Z ; M^{4}, F\right)$ is also (uniformly) continuous and, therefore, is continuous on the whole boundary $\mathrm{m}^{3}$.

THEOREM 5.2. Under conditions formulated previously on $M^{4}$, and additional conditions on $F(Z)$ to be formulated in $\$ 6$, there exist to every function $F(Z)$ and every lamina $I_{k}^{2}\left(\lambda_{k}\right)$, a B-harmonic function $U(Z)$ defined in $M^{4}$ such that

$$
\begin{array}{ll}
E\left(Z ; M^{4}, F\right)=U(Z) \text { for } \quad Z \in I_{k}^{2}\left(\lambda_{k}\right), \\
E\left(Z ; M^{4}, F\right) \leqq U(Z) \text { for } Z \in \bar{M}^{4} .
\end{array}
$$

We proceed now to the proof of the first theorem.

According to (2.5), every $i_{k}^{3}$ is a "cylinder" (or the sum of cylinders) which can be represented in the form

$$
z_{\kappa}=z_{\kappa}\left(Z_{k}, \lambda_{k}\right), \quad \quad \kappa=1,2 ; \lambda_{k} \in s_{k \alpha}^{1}, Z_{k} \in B_{k}^{2}\left(\lambda_{k}\right)
$$

where $s_{k \alpha}^{1}$ are connected intervals of the $\lambda_{k}$-axis, and $B_{k}^{2}\left(\lambda_{k}\right)=R^{-1}\left[I_{k}^{2}\left(\lambda_{k}\right)\right]$ are simply-connected domains in the $\mathbf{Z}_{k}$-plane.

As we shall show, the mapping $R_{\mathbf{k}}^{-1}$

$$
\begin{aligned}
& \left(z_{1}, z_{2}\right) \rightarrow\left(Z_{k}, \lambda_{k}\right), \\
& \left(z_{1}, z_{2}\right) \in i_{i \alpha \alpha}^{3}=\underset{\lambda_{k} \in s_{k \alpha}^{1}}{\mathcal{S}} I_{k}^{2}\left(\lambda_{k}\right), \quad\left(Z_{k}, \lambda_{k}\right) \in \underset{\lambda_{k} \in s_{k \alpha}^{1}}{\mathcal{S}} B_{k}^{2}\left(\lambda_{k}\right)
\end{aligned}
$$

is one-to-one and continuous. As follows from Lemma 5.1 (to be formulated and proved subsequently), the boundary of $d_{k \alpha}^{3}$ is $S_{\lambda_{k} \in s_{k \alpha}^{1}}^{1} b^{1}\left(\lambda_{k}\right)$, and 
$R_{\lambda_{k}}^{-1}\left[i_{k}^{1}\left(\lambda_{k}\right)\right]=b_{k}^{1}\left(\lambda_{k}\right)$ so that $b_{k}^{1}\left(\lambda_{k}\right)$ consists of images of $K$-points. The function $F(Z)$ given on the distinguished boundary surface is defined at every $K$-point and therefore at every point of $b_{k}^{1}\left(\lambda_{k}\right)$. Thus a real function is defined at every point of each $b_{k}^{1}\left(\lambda_{k}\right)$. Since by the hypothesis (2.9) each $b_{k}^{1}\left(\lambda_{k}\right)$ consists of finitely many rectifiable curves, we can for every $\lambda_{k}$ determine in $B_{k}^{2}\left(\lambda_{k}\right)=R_{\lambda_{k}}^{-1}\left[I_{k}^{2}\left(\lambda_{k}\right)\right]$ the harmonic function $U_{1}\left(Z_{k}, \lambda_{k}\right)$ which in $b_{k}^{1}\left(\lambda_{k}\right)$ $=R_{\lambda_{k}}^{-1}\left[i_{\mathbf{k}}^{1}\left(\lambda_{k}\right)\right]$ assumes the prescribed values $F\left[Z\left(Z_{k}, \lambda_{k}\right)\right], \mathbf{Z}_{k} \in b_{k}^{1}\left(\lambda_{k}\right)$. Since the values of $F[Z], Z \in i_{\boldsymbol{k}}^{1}\left(\lambda_{k}\right)$, and therefore those of $F\left[Z\left(\mathbf{Z}_{k}, \lambda_{k}\right)\right], \mathbf{Z}_{k} \in b_{k}^{1}\left(\lambda_{k}\right)$, when $\lambda_{k}$ varies, vary continuously, for every $\lambda_{k} \in s_{\boldsymbol{k}_{\alpha} \alpha}^{1},(2.7)$ holds, it follows by classical results that $U_{1}\left(\mathbf{Z}_{k}, \lambda_{k}\right)$ is a uniformly continuous function of $\mathrm{Z}_{k}, \lambda_{k},\left(\mathrm{Z}_{k}, \lambda_{k}\right) \in \bar{d}_{k \alpha}^{3}=S_{\lambda_{k} \in \bar{s}_{k \alpha}^{1}} \bar{B}_{k}^{2}\left(\lambda_{k}\right)$.

Since, further, the mapping $\boldsymbol{R}_{k}$ is one-to-one and continuous in every $\bar{d}_{k \alpha}^{3}$, and $\boldsymbol{R}_{k}\left(\bar{d}_{k \alpha}^{3}\right)=\bar{i}_{k \alpha}^{3}, U(Z)=U_{1}\left[\mathbf{Z}_{k}(Z), \lambda_{k}(Z)\right]$ is (uniformly) continuous in $\bar{i}_{k \alpha}^{3}$.

In order to establish Theorem 5.1 , it remains merely to prove the following lemma

LEMMA 5.1. The boundary $b_{k}^{1}\left(\lambda_{k}\right), \lambda_{k} \in s_{k \alpha}^{1}$ (or, what is the same, $i_{\mathbf{k}}^{1}\left(\lambda_{k}\right)$ since the mapping $\boldsymbol{R}_{\lambda_{k}}$ is one-to-one and continuous) changes continuously when $\lambda_{k}$ varies in the interval $\boldsymbol{s}_{\boldsymbol{k} \alpha}^{1}$.

Proof. By hypothesis (2.2) the boundary curves $j_{k}^{1}\left(\lambda_{k}\right)$ of each $Q_{k}^{2}\left(\lambda_{k}\right)$ vary continuously. Each $I_{k}^{2}\left(\lambda_{k}\right)$ represents a part of $Q_{k}^{2}\left(\lambda_{k}\right)$. If $i_{k}^{1}\left(\lambda_{k}\right)$ did not vary continuously, then the boundary $i_{k \alpha}^{3}=S_{\lambda_{k} \in s_{k a}^{2}} I_{k}^{2}\left(\lambda_{k}\right)$ would consist also of parts of some laminas $I_{k}^{2}\left(\lambda_{k}\right)$, that is, of $J$-points. We shall show that this is impossible.

Let $Z\left(\lambda_{k}\right) \equiv R_{k}\left(\mathrm{Z}_{\mathbf{k}}^{(1)}, \lambda_{k}\right), \mathrm{Z}_{\mathbf{k}}^{(1)}$ fixed, represent a sequence of points each of which lies on $Q_{k}^{2}\left(\lambda_{k}\right)$, and suppose that for $\lambda_{k}<\lambda_{k_{1}},\left(\lambda_{k_{1}}-\lambda_{k}\right)$ sufficiently small, $Z\left(\lambda_{k}\right)$ are $J$-points, that is, $Z\left(\lambda_{k}\right) \in I_{k}^{2}\left(\lambda_{k}\right)$. We shall show that the points $Z\left(\lambda_{k}\right) \equiv R_{k}\left(Z_{k}^{(1)}, \lambda_{k}\right), \lambda_{k}>\lambda_{k_{1}},\left(\lambda_{k}-\lambda_{k_{1}}\right)$ sufficiently small, must also be $J$-points. $R_{k}\left(Z_{k}^{(1)}, \lambda_{k_{1}}\right)$ is a point of $Q_{k}^{2}\left(\lambda_{k}\right)$, and therefore by (2.3) it is possible to construct a sufficiently small hypersphere $S^{4}$ with the center at $Z\left(\lambda_{k_{1}}\right)$ which does not include any $K$-points and such that $j_{k}^{3}$ divides $S^{4}$ into two parts. Let $\lambda_{k_{2}}$ be chosen so near $\lambda_{k_{1}}$ that the point $R_{k}\left(Z_{k}^{(1)}, \lambda_{k_{2}}\right), \lambda_{k_{2}}>\lambda_{k_{1}}$, lies in $S^{4}$. $R_{k}\left(Z_{k}^{(1)}, \lambda_{k_{2}}\right)$ can be either a boundary point of $M^{4}$ or an exterior point of $M^{4}$. The second alterrative is impossible, since we know that $S^{4}$ is divided by $j_{k}^{3}$ into two parts and all points of one part belong to the interior of $M^{4}$. Thus $\boldsymbol{R}_{k}\left(\mathbf{Z}_{k}^{(1)}, \lambda_{k_{2}}\right)$ belongs to the boundary of this part of $S^{4}$ and therefore in every neighborhood of $\boldsymbol{R}_{k}\left(\mathbf{Z}_{k}^{(1)}, \lambda_{k_{2}}\right)$ there are interior points of $M^{4}$. Consequently $R_{k}\left(Z_{k}^{(1)}, \lambda_{k_{2}}\right)$ is a boundary point of $M^{4}$, q.e.d.

6. The proof of Theorem 5.2. In this section we shall give the proof of Theorem 5.2, that is, we shall show that to every function $F(Z)$ given on the distinguished boundary surface $M^{2}$ and satisfying certain conditions, there exists a $B$-harmonic function $U(Z)$ of the class $U(F, M)$ (see p. 427) which coincides on the boundary curve $i_{k}^{1}\left(\lambda_{k}\right)$ of a given lamina $I^{2}=I_{k}^{2}\left(\lambda_{k}\right)$ with 
$F(Z)$. (It is to be noted that $i_{k}^{1}\left(\lambda_{k}\right)=I_{k}^{2}\left(\lambda_{k}\right) \cap M^{2}$.) If therefore $z_{1}^{*}$ and $z_{2}^{*}$ denote coordinates which are normal with respect to the lamina $I^{2}$,

$$
\begin{aligned}
F\left[Z\left(0,0 ; x_{2}^{*}, y_{2}^{*}\right)\right] & =U\left[Z\left(0,0 ; x_{2}^{*}, y_{2}^{*}\right)\right], & \left(x_{2}^{*}, y_{2}^{*}\right) & \in i^{* 1} . \\
F(Z) & \leqq U(Z), & Z & \in M^{2} .
\end{aligned}
$$

In order to prove uur statement we have, however, to make some additional hypotheses on $F$.

(6.1) Let $h\left(z_{2}^{*}\right)=S\left(x_{2}^{*}, y_{2}^{*}\right)^{\top}+i T\left(x_{2}^{*}, y_{2}^{*}\right)$ be that analytic function whose real part $S$ assumes the values of $F\left(0,0 ; x_{2}^{*}, y_{2}^{*}\right)$ on the boundary $i^{* 1}$ of $I^{* 2} \equiv I_{k}^{* 2}\left(\lambda_{k}\right)$. We assume that there exists a constant $\rho^{\prime \prime}$ such that for every point $\left(x_{2}^{*(0)}, y_{2}^{*(0)}\right) \in i^{* 1}, S$ and $T$ can be represented in the intersection of $\bar{I}^{* 2}$ and the circle with center $\left(x_{2}^{*(0)}, y_{2}^{*(0)}\right)$ and the radius $\rho^{\prime \prime}$ in the form

$$
S\left(x_{2}^{*}, y_{2}^{*}\right)=\alpha^{(0)}+\left(x_{2}^{*}-x_{2}^{*(0)}\right) \alpha_{1}^{(0)}+\left(y_{2}^{*}-y_{2}^{*(0)}\right) \alpha_{2}^{(0)}, \alpha_{k}^{(0)}=\alpha_{k}^{(0)}\left(x_{2}^{*}, y_{2}^{*}\right),
$$

$$
\begin{gathered}
\alpha^{(0)}=S\left(x_{2}^{*(0)}, y_{2}^{*(0)}\right) \equiv F\left[Z\left(0, z_{2}^{*(0)}\right)\right], \\
T\left(x_{2}^{*}, y_{2}^{*}\right)=\beta^{(0)}+\left(x_{2}^{*}-x_{2}^{*(0)}\right) \beta_{1}^{(0)}+\left(y_{2}^{*}-y_{2}^{*(0)}\right) \beta_{2}^{(0)}, \beta_{k}^{(0)}=\beta_{k}^{(0)}\left(x_{2}^{*}, y_{2}^{*}\right), \\
\beta^{(0)}=T\left(x_{2}^{*(0)}, y_{2}^{*(0)}\right),
\end{gathered}
$$

where there exists a constant $\gamma$ independent of $x_{2}^{*(0)}, y_{2}^{*(0)}$ such that for $x_{2}^{*}, y_{2}^{*}$ in the given circle

$$
\left|\alpha^{(0)}\right|,\left|\beta^{(0)}\right|,\left|\alpha_{k}^{(0)}\right|,\left|\beta_{k}^{(0)}\right|<\gamma, \quad \kappa=1,2 .
$$

(6.2) We assume that $F(Z)$ satisfies a Lipschitz condition, that is, if $Z^{*(\kappa)}\left(z_{1}^{*(\kappa)}, z_{2}^{*(\kappa)}\right) \in M^{2}, \kappa=1,2$, then

$$
\begin{aligned}
\left|F\left[Z\left(Z^{*(1)}\right)\right]-F\left[Z\left(Z^{*(2)}\right)\right]\right| \leqq & C\left(\left|z_{1}^{*(1)}-z_{1}^{*(2)}\right|^{1 / x}\right. \\
& \left.+\left|z_{2}^{*(1)}-z_{2}^{*(2)}\right|^{1 / x}\right)
\end{aligned}
$$

where $C$ and $\chi$ are suitably chosen constants.

We finally note that it is no restriction to assume that

$$
4 a \geqq F(Z) \geqq 2 a, a \geqq\left|T\left(x_{2}^{*}, y_{2}^{*}\right)\right| .
$$

This is because a fixed constant is a $B$-harmonic function and can be added and subtracted from $S$ and $F$ without changing $T$.

We proceed to the construction of the function $U\left(Z^{*}\right)$. The function

$$
S\left[\operatorname{Re}\left(z_{2}^{*}\left(1-\sigma z_{1}^{* 1 / \mu}\right)\right), \operatorname{Im}\left(z_{2}^{*}\left(1-\sigma z_{1}^{* 1 / \mu}\right)\right)\right]
$$

$$
+i T\left[\operatorname{Re}\left(z_{2}^{*}\left(1-\sigma z_{1}^{* 1 / \mu}\right)\right), \operatorname{Im}\left(z_{2}^{*}\left(1-\sigma z_{1}^{* 1 / \mu}\right)\right)\right],
$$


where $\sigma$ and $\mu$ are constants introduced in Theorem 3.1, is defined in the domain $A^{4}$ and therefore in $M^{4}$. Since the points $\left(z_{1}^{*}, z_{2}^{*}\right)$ lying on the negative real axis $\mathrm{E}\left[x_{2}^{*}<0, y_{2}^{*}=0\right]$ do not belong to $M^{4}, z_{1}{ }^{* 1 / \mu}$ is uniquely defined for any point $Z^{*}$ by the assumption that

$$
-\pi<\arg z_{1}^{*}<\pi \text {. }
$$

We now multiply the function (6.8) by $\left(1+k z_{1}{ }^{* 1 / \nu}\right), k>0, \nu>0$, and write

$$
U^{*}=\operatorname{Re}\left\{[S+i T]\left[1+k z_{1}^{* 1 / v}\right]\right\} .
$$

It is apparent that $U^{*}$ coincides with $F$ on $i_{k}^{* 1}\left(\lambda_{k}\right)$. We shall show that $k, k>1$, and $\nu$ can be chosen large enough so that $U^{*}\left(Z^{*}\right) \geqq F\left[Z\left(Z^{*}\right)\right], Z^{*} \in M^{* 2}$.

We first consider that part of $M^{* 2}$ for which $\left|z_{1}^{*}\right| \leqq \rho \leqq \min \left(\rho^{\prime}, \rho^{\prime \prime}\right)$. (Concerning $\rho^{\prime}$ see (3.8).) Consider an arbitrary point $\left(z_{1}^{*}, z_{2}^{*}\right)$ of $M^{* 2}$, such that $\left|z_{1}^{*}\right| \leqq \rho$ and let $z_{2}^{*(0)}=x_{2}^{*(0)}+i y_{2}^{*(0)}$ be the point defined in hypothesis (3.8). Let us denote $z_{2}^{*}-z_{2}^{*(0)}$ by $\tilde{z}_{2}^{*}=\tilde{x}_{2}^{*}+i \tilde{y}_{2}^{*}$. We shall show that by choosing $\rho$ sufficiently small and $\nu$ sufficiently large, both independently of $k \geqq 1$, we can insure that

$$
U^{*}\left(Z^{*}\right) \geqq F\left[Z\left(Z^{*}\right)\right], \quad Z^{*} \in M^{* 2}, \quad\left|z_{1}^{*}\right| \leqq \rho .
$$

Now by hypothesis (6.1)

$$
\begin{array}{r}
S \equiv S\left[x_{2}^{*}\left(1-\sigma r_{1}^{1 / \mu} \cos \frac{\phi_{1}}{\mu}\right)+y_{2}^{* \sigma r_{1}^{1 / \mu}} \sin \frac{\phi_{1}}{\mu}, y_{2}^{*}\left(1-\sigma r_{1}^{1 / \mu} \cos \frac{\phi_{1}}{\mu}\right)\right. \\
\left.-x_{2}^{* \sigma r_{1}^{1 / \mu}} \sin \frac{\phi_{1}}{\mu}\right] \geqq \alpha^{(0)}-\gamma\left|L_{1}\right|-\gamma\left|L_{2}\right|
\end{array}
$$

where

$$
\begin{aligned}
& L_{1}=\tilde{x}_{2}^{*}-x_{2}^{*} \sigma r_{1}^{1 / \mu} \cos \frac{\phi_{1}}{\mu}+y_{2}^{*} \sigma r_{1}^{1 / \mu} \sin \frac{\phi_{1}}{\mu}, \\
& L_{2}=\tilde{y}_{2}^{*}-x_{2}^{*} \sigma r_{1}^{1 / \mu} \cos \frac{\phi_{1}}{\mu}-x_{2}^{*} \sigma r_{1}^{1 / \mu} \sin \frac{\phi_{1}}{\mu} .
\end{aligned}
$$

We may now use hypothesis (3.7) to estimate $L_{1}$ and $L_{2}$. By (3.8) it follows that $\left|\tilde{x}_{2}^{*}\right| \leqq A r_{1}^{1 / \tau},\left|\tilde{y}_{2}^{*}\right| \leqq A r_{1}^{1 / \tau}$. Here $\tau=\max \left[\tau^{\prime}, \mu\right]$, see $(3.23), r_{1}=\left|z_{1}^{*}\right|$. Trivially one obtains

$$
S \geqq \alpha^{(0)}-R_{r_{1}}^{1 / \tau}, \quad R=6 A \gamma .
$$

By construction

$$
|T| \leqq a \text {. }
$$

We now recall the definition of $U^{*}$ and obtain immediately on assuming 
that $\nu$ is sufficiently large so that $\cos (\pi / \nu)>2 / 3, \sin (\pi / \nu)<1 / 3$,

$$
U^{*} \geqq\left[\alpha^{(0)}-R r_{1}^{1 / \tau}\right]\left[1+2 k r_{1}^{1 / \nu} / 3\right]-a\left[k r_{1}^{1 / \nu} / 3\right] .
$$

Recalling that $\alpha^{(0)} \equiv F\left[Z\left(0, z_{2}^{*(0)}\right)\right] \geqq 2 a$, we obtain

$$
U^{*} \geqq \alpha^{(0)}+a k r_{1}^{1 / \nu}-R(1+k) r_{1}^{1 / \tau} \text {. }
$$

Simultaneous we have, using (6.2), that

$$
\left|F\left[Z\left(z_{1}^{*}, z_{2}^{*}\right)\right]-F\left[Z\left(0, z_{2}^{*(0)}\right)\right]\right| \leqq C\left(\left|z_{1}^{*}\right|^{1 / x}+\left|\tilde{z}_{2}^{*}\right|^{1 / x}\right),
$$

that is, $F\left[Z\left(z_{1}^{*}, z_{2}^{*}\right)\right] \leqq \alpha^{(0)}+C r_{1}^{1 / x}+A C r_{1}^{1 / \tau x}$. We shall certainly assure $U^{*} \geqq F$ if

$$
a k r_{1}^{1 / \nu} \geqq R(1+k) r_{1}^{1 / \tau}+C r_{1}^{1 / x}+A C r_{1}^{1 / \tau x},
$$

and this will be true if

$$
k r_{1}^{1 / v} \geqq k R^{\prime} r_{1}^{1 / \tau x} \text { where } R^{\prime}=[2 R+C+A C] / a,
$$

that is, if $r_{1}^{1 / v} \geqq R_{1}^{1 / \tau x}$. We may pick $\rho$ so small and $\nu$ so large that $\left({ }^{26}\right)$

$$
r_{1}^{1 / \nu} \geqq R^{\prime} r_{1}^{1 / \tau x} \quad \text { for } r_{1}<\rho .
$$

Our proof will now be complete if we can show that a proper choice of $k$ will insure $U^{*}\left(Z^{*}\right) \geqq F\left[Z\left(Z^{*}\right)\right]$ for $Z^{*} \in M^{* 2},\left|z_{1}^{*}\right| \geqq \rho$. Using that $S \geqq 2 a$, $|T|<a, \cos (\pi / \nu)>2 / 3, \sin (\pi / \nu)<1 / 3$, we have

$$
\begin{aligned}
U^{*} & \geqq 2 a\left[1+2 k r_{1}^{1 / \nu} / 3\right]-a\left[k r_{1}^{1 / \nu} / 3\right] \\
& =2 a+a k r_{1}^{1 / \nu}=a\left[2+k r_{1}^{1 / \nu}\right] .
\end{aligned}
$$

But $F \leqq 4 a$. We need merely choose $k$ so large that $k \rho^{1 / \nu}>2$.

Since an analogous procedure may be applied to obtain a function of the class $\mathcal{L}\left(F, M^{4}\right)$, we obtain the following:

THEOREM 6.1. To every function $F(Z)$ defined on $M^{2}$ and satisfying conditions 6.1-6.2 and to every lamina $I_{k}^{2}\left(\lambda_{k}\right)$ there exist B-harmonic functions $u(Z)$ and $l(Z)$ of the classes $U(F, M)$ and $\mathcal{L}(F, M)$, respectively, such that on $I_{k}^{2}\left(\lambda_{k}\right)$ we have

$$
u(Z)=l(Z)=h\left[x_{2}^{*}(Z), y_{2}^{*}(Z)\right], \quad Z \in I_{k}^{2}\left(\lambda_{k}\right),
$$

where $h\left[x_{2}^{*}, y_{2}^{*}\right]$ is that harmonic function which on $i_{k}^{1}\left(\lambda_{k}\right)$ assumes the values $F\left[Z\left(0, z_{2}^{*}\right)\right]$.

REMARK. By a slight modification of the proof it is possible to replace (6.1), (6.2) by weaker hypotheses; it suffices (essentially) to assume that $F$ is continuous.

(26) For example choosing $\nu=2 \tau \chi$ and $\rho \leqq\left(1 / R^{\prime}\right)^{2 \tau x}$. 
7. Determination in $M^{4}$ of functions $E h\left(Z ; M^{4} ; F\right)$ of extended class and some of their properties. By the procedure indicated in $\$ 5$, we determine in every lamina $I_{k}^{2}\left(\lambda_{k}\right)$ a real function. Since the whole boundary $m^{3}$ of $M^{4}$ can be represented as a sum of laminas, a real function is defined in $m^{3}$.

If $F(Z)$ is (uniformly) continuous in $M^{2}$, then by Theorem 5.1 this function is (uniformly) continuous in $m^{3}$, and assumes the values $F(Z)$ if we approach in $m^{3}$ the distinguished boundary surface $M^{2}$.

As we indicated in $\$ 1$, there are two possibilities of extending the function throughout $M^{4}$.

The first possibility consists in constructing that harmonic function

$$
E h\left[Z ; M^{4} ; F\right], \quad Z \in M^{4},
$$

which assumes on $m^{3}$ the value determined previously.

If $M^{4}$ satisfies hypothesis 4.1 , then it is known from the theory of potential that to every (uniformly) continuous function defined on $m^{3}$ there exists a harmonic function of four variables defined in $M^{4}$ which assumes the prescribed values on $m^{8}$. Thus we obtain:

THEOREM 7.1. If the domain $M^{4}$ satisfies the conditions formulated in $\$ \S 2$, 3 , and 4 , then to every function $F(Z)$ defined on the distinguished boundary surface $M^{2}$ and satisfying hypotheses 6.1-6.2, there exists one and only one function $E h\left[Z ; M^{4} ; F\right]$ of extended class which assumes on $M^{2}$ the values $F(Z)$.

From the definition of the functions $E h\left[Z ; M^{4} ; F\right]$ follow almost immediately the following lemmas:

LEMMA 7.1. If

$$
F_{1}(Z) \geqq F_{2}(Z), \quad Z \in M^{2},
$$

then

$$
E h\left[Z ; M^{4} ; F_{1}\right] \geqq E h\left[Z ; M^{4} ; F_{2}\right], \quad Z \in \bar{M}^{4} .
$$

Lemma 7.2. If $B(Z)$ is $B$-harmonic in $M^{4}$ and satisfies on $M^{2}$ the hypotheses 6.1-6.2, then

$$
E h\left[Z ; M^{4} ; B\right]=B(Z), \quad Z \in \bar{M}^{4} .
$$

From Lemmas 7.1 and 7.2 follows:

Corollary 7.1. If $B_{k}(Z)(\kappa=1,2)$ are B-harmonic functions which satisfy on $M^{2}$ the hypotheses 6.1-6.2, and such that

$$
(-1) \times F(Z) \geqq B_{\kappa}(Z), \quad Z \in M^{2},
$$

then

$$
E h\left[Z ; M^{4} ;(-1)^{\wedge} F\right] \geqq B_{\kappa}(Z), \quad Z \in M^{4}
$$


Lemma 7.3. If $M^{4}=E\left[\left|z_{1}\right|<r_{1},\left|z_{2}\right|<r_{2}\right]$, that is, if $M^{4}$ is a bicylinder and $F(Z)$ satisfies hypotheses 6.1-6.2, then $E h\left[Z ; M^{4}: F\right]$ is that doubly harmonic function which assumes on $M^{2}=\mathrm{E}\left[z_{1}\left|=r_{1},\right| z_{2} \mid=r_{2}\right]$ the values $F(Z)$.

8. Properties of functions $E n\left[Z ; F ; M^{4}\right]$ in $M^{4}$. In this section we shall indicate how to determine the values of $E_{n}\left[Z: F ; M^{4}\right]$ in the interior of $M^{4}$ and we shall show that the hypotheses (4.1a)-(4.3a) insure that a theorem analogous to 7.1 [proved in $\S 7$ for $E h\left[Z ; F ; M^{4}\right]$ ] holds for the $E n\left[Z ; F ; M^{4}\right]$.

We begin by proving that the functions $E n\left[Z ; F ; M^{4}\right]$ are continuous. Let $\left(z_{1}^{(0)}, z_{2}^{(0)}\right)$ be an inner point of $M^{4}$. Then $z_{2}^{(0)}$ must be an inner point of the projection $P^{2}$, see (4.1a); for, if this were not the case, then in every neighborhood of $z_{2}^{(0)}$ there would exist points $z_{2}^{(s)} \in P^{2}$, such that $M^{4}\left[z_{2}=z_{2}^{(s)}\right]$ were empty, and in every neighborhood of $\left(z_{1}^{(0)}, z_{2}^{(0)}\right)$ we would have points $\left(z_{1}^{(0)}, z_{2}^{(8)}\right)$ which do not belong to $M^{4}$. Therefore $\left(z_{1}^{(0)}, z_{2}^{(0)}\right)$ would not be an inner point of $M^{4}$.

Since $F\left(z_{1}, z_{2}^{(0)}\right)$ is a continuous function of $z_{1}$ on the boundary of $M^{4}\left(z_{2}=z_{2}^{(0)}\right)$, it follows from (4.2a) that $E n\left[z_{1}, z_{2}^{(0)} ; F ; M^{4}\right]$ is a continuous function of $z_{1}$ in $M^{4}\left(z_{2}=z_{2}^{(0)}\right)$. The function $E n\left[Z ; F ; M^{4}\right]$ is continuous on $m^{3}$, and every $N_{\alpha}^{2}\left(z_{2}^{(0)}\right)$ has the property that to every interior point $\left(z_{1}^{(0)}, z_{2}^{(0)}\right) \in M^{4}$ belonging to any $N_{\alpha}^{2}\left(z_{2}^{(0)}\right)$ there exists a neighborhood $\left|z_{2}^{(0)}-z_{2}\right| \leqq \epsilon$ such that for $\left|z_{2}^{(0)}-z_{2}\right| \leqq \epsilon$ the boundary of $N_{\alpha}^{2}\left(z_{2}^{(0)}\right)$ varies continuously, and $N_{\alpha}^{2}\left(z_{2}^{(0)}\right)$ is the kernel domain of the sequence of domains $N_{\alpha}^{2}\left(z_{2}\right)$, for $z_{2} \rightarrow z_{2}^{(0)}$. Therefore, from (uniform) continuity on the boundaries of the $N_{\alpha}^{2}\left(z_{2}\right)$ of $E n\left[Z ; F, M^{4}\right]$ considered as function of $z_{2}$ follows the (uniform) continuity in $N_{\alpha}^{2}\left(z_{2}\right)$, for $\left|z_{2}-z_{2}^{(0)}\right| \leqq \epsilon$. Thus $E n\left[Z ; F, M^{4}\right]$ is (uniformly) continuous considered as function of $z_{1}, z_{2}$ at every point of $M^{4}$. Since further it is uniformly continuous considered as function of $z_{1}$ in every $N_{\alpha}^{2}\left(z_{2}\right)$, and since $E n\left[Z ; F, M^{4}\right]$ is (uniformly) continuous on the boundary, it follows that $\operatorname{En}\left[Z ; F ; M^{4}\right]$ is (uniformly) continuous in $\bar{M}^{4}$.

In particular, it follows from this that $\lim _{Z \rightarrow Z^{(0)}} E n\left[Z ; M^{4} ; F\right]=F\left(Z^{(0)}\right)$ for $Z^{(0)} \in M^{2}$.

Until the present we assumed that $F(Z)$ is a (uniformly) continuous function defined on $M^{2}$. It is, however, comparatively easy to generalize our considerations to the case where $F(Z)$ is continuous on $M^{2}$, except possibly on a curve $n^{1}$, where it has a finite jump.

9. Inequalities for bounded analytic functions of two complex variables. Ostrowski's two constants theorem is one of the most simple applications of the principle of harmonic measure. The introduction of functions of extended class enables us to generalized this result.

Notation. The functions $E h\left[Z ; M^{4}, m\right]$, where

$$
\text { (9.1) } m(Z)=1 \text { for } Z \in M_{1}^{2} \subset M^{2}, \quad m(Z)=0 \text { for } Z \in M^{2}-M_{1}^{2} \text {, }
$$

will be called (in generalizing the classical concept) the $E h$-harmonic measure 
of the segment $M_{1}^{2}$, and will be denoted by

$$
E h\left[Z ; M^{4} ; M_{1}^{2}\right] .
$$

THEOREM 9.1. Let $g\left(z_{1}, z_{2}\right)$ be an analytic function of two complex variables which is regular in $M^{4}$, and for which

$$
\begin{array}{lr}
\left|g\left(z_{1}, z_{2}\right)\right| \leqq a<1, & \left(z_{1}, z_{2}\right) \in M_{1}^{2} \subset M^{2}, \\
\left|g\left(z_{1}, z_{2}\right)\right| \leqq 1, & \left(z_{1}, z_{2}\right) \subset M^{2},
\end{array}
$$

holds. Then

$$
\left|g\left(z_{1}, z_{2}\right)\right| \leqq \exp \left(\lg a \times E h\left[Z ; M^{4} ; M_{1}^{2}\right]\right) .
$$

Proof. Since $g\left(z_{1}, z_{2}\right)$ is an analytic function of two complex variables, $\lg \left|g\left(z_{1}, z_{2}\right)\right|$ is a $B$-harmonic function which is regular in $M^{4}$, except perhaps at a finite number of analytic surfaces [zero surfaces of $g\left(z_{1}, z_{2}\right)$ ] where $\lg \left|g\left(z_{1}, z_{2}\right)\right|$ becomes logarithmically $-\infty$. We shall at first show that the inequality

$$
\lg \left|g\left(z_{1}, z_{2}\right)\right| \leqq \lg a \times E h\left[Z ; M^{4} ; M_{1}^{2}\right]
$$

holds in every lamina, say $I_{k}^{2}\left(\lambda_{k}\right)$.

Two cases can occur: (1) $I_{k}^{2}\left(\lambda_{k}\right)$ is a zero surface of $g\left(z_{1}, z_{2}\right)$. Then $\lg \left|g\left(z_{1}, z_{\varepsilon}\right)\right|=-\infty$ for $\left(z_{1}, z_{2}\right) \in I_{k}^{2}\left(\lambda_{k}\right)$, and therefore the inequality (9.6) holds there.

(2) If $I_{k}^{2}\left(\lambda_{k}\right)$ is not a zero surface of $g\left(z_{1}, z_{2}\right)$, then $g\left(z_{1}, z_{2}\right)$ can vanish there only at a finite number of points. $\lg \left|g\left(z_{1}, z_{2}\right)\right|$ and $E h\left[Z ; M^{4} ; M_{1}^{2}\right]$ are harmonic functions of real variables $\operatorname{Re} Z_{k}, \operatorname{Im} Z_{k}$. Since $i_{k}^{1}\left(\lambda_{k}\right) \subset M^{2}$, by assumption the inequality (9.6) holds on $i_{k}^{1}\left(\lambda_{k}\right)$, and therefore by the classical result of the theory of harmonic functions of two real variables, also for $\left(z_{1}, z_{2}\right)$ $\in I_{k}^{2}\left(\lambda_{k}\right)$. Thus (9.6) holds in every $I_{k}^{2}\left(\lambda_{k}\right)$ and therefore in the whole $m^{3}$.

We proceed now to establish (9.6) for $Z \in M^{4}$. Eh $\left[Z ; M^{4} ; M_{1}^{2}\right]$ and $\lg \left|g\left(z_{1}, z_{2}\right)\right|$ are harmonic functions of four real variables. (We note that the latter function becomes $-\infty$ along the zero surfaces of $g\left(z_{1}, z_{2}\right)$, if such exist.) According to the classical results of the theory of harmonic functions of four real variables, the validity of the inequality (9.6) on the boundary of the domain implies the same inequality for points inside the domain $M^{4}$, q.e.d.

Notation. In analogy to $(9.2)$ we shall denote $E n\left[Z ; M^{4} ; m\right]$ by

$$
E n\left[Z ; M^{4} ; M_{1}^{2}\right]
$$

and shall call it $E n$-harmonic measure of the segment $M_{1}^{2}$.

Corollary 9.1. Under the conditions as in Theorem 9.1, there holds:

$$
\left|g\left(z_{1}, z_{2}\right)\right| \leqq \exp \left(\lg a \times E n\left[Z ; M^{4} ; M_{1}^{2}\right]\right) .
$$


Proof. On $m^{3}$,

$$
E n\left[Z ; M^{4} ; M_{1}^{2}\right]=E h\left[Z ; M^{4} ; M_{1}^{2}\right]
$$

and as before we obtain that on every $m^{3}\left(z_{2}=z_{2}\right), \lg a \times E n\left[Z ; M^{4} ; M_{1}^{2}\right]$ $\geqq \lg \left|g\left(z_{1}, z_{2}\right)\right|$. In $M^{4}\left(z_{2}=z_{2}\right), E\left[Z ; M^{4} ; M_{1}^{2}\right]$ as well as $\log \mid g\left(z_{1}, z_{2}\right)$ are harmonic functions of the variables $\operatorname{Re} Z, I m Z$, see II p. 526 and (4.1), $E\left[Z ; M^{4} ; M_{1}^{2}\right]$ is a regular harmonic function, while $\log \left|g\left(z_{1}, z_{2}\right)\right|$ is regular except perhaps at a finite number of zero points of function $g\left[n_{1}\left(\mathrm{Z}, \mu_{1}, \mu_{2}\right)\right.$, $\left.n_{2}\left(\mathrm{Z}, \mu_{1}, \mu_{2}\right)\right]=g\left[\mathrm{Z}, \mu_{1}+i \mu_{2}\right], \mu_{1}+i \mu_{2}=z_{2}=$ fixed, where $\log \left|g\left[\mathrm{Z}, \mu_{1}+i \mu_{2}\right]\right|$ becomes $-\infty$. From the validity of (9.9) on the boundary of $M^{4}\left(z_{2}=z_{2}\right)$, it follows that the same inequality holds inside $M^{4}\left(z_{2}=z_{2}\right)$, q.e.d.

\section{BIBLIOGRAPHY}

1. Stefan Bergman, Über die Kernfunktion eines Bereiches und ihr Verhalten am Rande, J. Reine Angew. Math. vol. 169 (1933) pp. 1-42, and vol. 172 (1934) pp. 89-128.

2. - Über eine in gewissen Bereichen mit Maximumfäche gilltige Integralderstellung der Funktionen zweier komplexer Variabler, Math. Zeit. vol. 39 (1935) pp. 76-94 and 605-608.

3. - U Über eine Integraldarstellung von Funktionen zweier komplexer Veränderlichen, Rec. Math. (Mat. Sbornik) N. S. vol. 1 (1936) pp. 851-862.

4. - Über meromorphe Funktionen von zwei komplexen Veränderlichen, Compositio Math. vol. 6 (1939) pp. 305-335.

5. - Theory of pseudo-conformal transformations and its connection with differential geometry, Notes on lectures delivered at the Massachusetts Institute of Technology, 1939-1940 (available at the Brown University library).

6. - On the surface integrals of functions of two complex variables, Amer. J. Math. vol. 63 (1941) pp. 295-318.

7. —, Über uneigentliche Flächenintegrale in der Theorie der analytischen Funktionen von zwei komplexen Veranderlichen, Revista de Ciencias, Universidad Nacional Mayor de San Marcos, Lima, Peru, no. 438 (1941) pp. 675-682, no. 439 (1942) pp. 131-140, no. 441 (1942) pp. 377-394.

8. - The behavior of the kernel function at boundary points of the second order, Amer. J. Math. vol. 65 (1943) pp. 679-700.

9. S. Bergman and M. Schiffer, Bounded functions of two complex variables, Amer. J. Math. vol. 66 (1944) pp. 161-169.

10. Lipman Bers, On bounded analytic functions of two complex variables in certain domain with distinguished boundary surface, Amer. J. Math. vol. 64 (1942) pp. 514-530.

11. Abe Gelbart, On the growth properties of a function of two complex variables given by its power series expansion, Trans. Amer. Math. Soc. vol. 49 (1941) pp. 199-210.

HARVARD UNIVERSITY,

Cambridge, Mass. 